\title{
THE FUNDAMENTAL GROUP OF RANDOM 2-COMPLEXES
}

\author{
ERIC BABSON, CHRISTOPHER HOFFMAN, AND MATTHEW KAHLE
}

\section{INTRODUCTION}

In this article we find the threshold for simple connectivity of the random 2dimensional simplicial complexes $Y(n, p)$ introduced by Linial and Meshulam [10] to be roughly $p=n^{-1 / 2}$. One motivation for this is continuing the thread of probabilistic topology initiated by Linial and Meshulam [10, and even earlier by Erdös and Rényi [3]. (Other recent work concerning the topology of random simplicial complexes can be found in [8, 9, 11, 14.)

Another motivation for this study is the connection to the random groups studied in geometric group theory [12. In face we must use geometric group theory techniques to show that in the sparse regime the fundamental group is hyperbolic on the way to showing that it is nontrivial; in particular, we apply Gromov's localto-global principle for linear isoperimetric inequalities.

Erdős and Rényi initiated the now vast subject of random graphs with their edge-independent model $G(n, p)[3$.

Definition 1.1. The Erdös-Rényi random graph $G(n, p)$ is the probability space of all graphs on the vertex set $[n]:=\{1,2, \ldots, n\}$ with each of the $\left(\begin{array}{l}n \\ 2\end{array}\right)$ possible edges included independently with probability $p$. We say that $G(n, p)$ asymptotically almost surely (a.a.s.) has property $\mathcal{P}$ if $\lim _{n \rightarrow \infty} \mathbf{P}(G(n, p) \in \mathcal{P})=1$.

A seminal result is that $p=\log n / n$ is a sharp threshold for the connectivity of the random graph.

Theorem 1.2 (Erdős and Rényi [3]). Let $\omega(n) \rightarrow \infty$ as $n \rightarrow \infty$.

(1) If $p=(\log n-\omega(n)) / n$, then $G(n, p)$ is a.a.s disconnected, and

(2) if $p=(\log n+\omega(n)) / n$, then $G(n, p)$ is a.a.s. connected.

Nathan Linial and Roy Meshulam exhibited a 2-dimensional homological analogue of Theorem 1.2. They defined a model of random 2-dimensional simplicial complexes $Y(n, p)$ to be the probability space of simplicial complexes on the vertex set $[n]$ and edge set $\left(\begin{array}{c}{[n]} \\ 2\end{array}\right)$, with each 2 -face appearing independently with probability $p$.

Received by the editors November 7, 2008 and, in revised form, July 9, 2010.

2010 Mathematics Subject Classification. Primary 20F65; Secondary 05C80.

Key words and phrases. Random groups, hyperbolic groups.

The second author was supported in part by NSA grant \#H98230-05-1-0053 and NSF grant \#DMS-0501102 and by an AMS Centennial Fellowship.

The third author was supported in part by the University of Washington's NSF-VIGRE grant \#DMS-0354131.

We would also like to thank MSRI and the Institute for Advanced Studies at the Hebrew University of Jerusalem where some of the research was done.

(C)2010 American Mathematical Society Reverts to public domain 28 years from publication 
Theorem 1.3 (Linial-Meshulam [10). Let $\omega(n) \rightarrow \infty$ as $n \rightarrow \infty$. If $p=(2 \log n-$ $\omega(n)) / n$, then a.a.s. $H_{1}(Y, \mathbb{Z} / 2 \mathbb{Z}) \neq 0$, and if $p=(2 \log n+\omega(n)) / n$, then a.a.s. $H_{1}(Y, \mathbb{Z} / 2 \mathbb{Z})=0$.

Meshulam and Wallach extended this result to $H_{d-1}(Y, \mathbb{Z} / q \mathbb{Z})$ for arbitrary primes $q$ and $d$-dimensional complexes [11.

Our first result is that when $p$ is sufficiently large, $\pi_{1}(Y(n, p))$ a.a.s. vanishes.

Theorem 1.4. Let $\omega(n) \rightarrow \infty$ as $n \rightarrow \infty$. If

$$
p \geq\left(\frac{3 \log n+\omega(n)}{n}\right)^{1 / 2},
$$

then a.a.s. $\pi_{1}(Y)=0$.

Our main result and most of the work of this paper is to show that the exponent $1 / 2$ in Theorem 1.4 is the best possible.

Theorem 1.5. For any constant $\epsilon>0$, if

$$
p=O\left(\frac{n^{-\epsilon}}{n^{1 / 2}}\right)
$$

then $\pi_{1}(Y(n, p))$ is a.a.s. hyperbolic and nontrivial.

The proof of Theorem 1.5 relies on general notions of negative curvature due to Gromov. As the Linial-Meshulam result is an analogue of the Erdős-Rényi theorem, our result is analogous to certain thresholds for random groups. The random group seemingly closest to what we study here is the following triangular model.

Definition 1.6. Let $0 \leq d \leq 1$. A triangular random group on $n$ relators at density $d$ is the group presented by $H=\left\langle b_{1}, \ldots, b_{n} \mid R\right\rangle$, where $R=\left\{r_{1}, r_{2}, \ldots, r_{t}\right\}$, and each $r_{i}$ is chosen i.i.d. uniformly from the $T=2 n(2 n-1)^{2}$ reduced words of length 3 , and $t=\left\lfloor T^{d}\right\rfloor$.

Żuk characterized the threshold for vanishing of $H$ as $n \rightarrow \infty$.

Theorem 1.7 ([15]). If $d<1 / 2$, then $H$ is a.a.s. nontrivial hyperbolic, and if $d>1 / 2$, then $H$ is a.a.s. trivial.

Theorem 1.7 is similar in spirit to Theorem 1.5, but the proof of Theorem 1.5 seems to require new methods. We prove some intermediate results which may be of independent interest. In particular, we classify the homotopy type of simplicial complexes with a sparsity of faces. See Section 3.1 for the notation.

Theorem 1.8. If $X$ is a finite 2-dimensional simplicial complex such that

$$
2 f_{0}(W)>f_{2}(W)
$$

for all subcomplexes $W \subset X$, then $X$ has the homotopy type of a wedge of circles, spheres and real projective planes. Thus the fundamental group of $X$ is a free product of $\mathbb{Z}$ 's and $\mathbb{Z} / 2 \mathbb{Z}$ 's.

We use this to obtain a linear isoperimetric inequality for null-homotopic loops in $X$. (We precisely define cycles $\gamma$ and the notions of length $L(\gamma)$ and area $A(\gamma)$ on page 4 in Section 3 , ) 
Theorem 1.9. For any $\epsilon>0$ there exists $\beta(\epsilon)>0$ such that if $X$ is a finite, 2-dimensional simplicial complex with

$$
(2-\epsilon) f_{0}(W)>f_{2}(W)
$$

for all subcomplexes $W \subset X$, then every contractible cycle $\gamma$ satisfies

$$
L(\gamma)>\beta(\epsilon) A(\gamma)
$$

We also need a version of Gromov's general principle that one can go from local linear isoperimetric inequalities to global ones [5], a method which has been very useful in the study of random groups.

The rest of the paper is organized as follows. Section 2 contains the proof of Theorem [1.4. Section 3 contains the outline of the proof of Theorem [1.5. In Section 4 we prove Theorem 1.8. We use this in Section 5 to prove Theorems 1.5 and 1.9. Section 6 discusses open problems and further connections of this work with geometric group theory. The appendices prove a technical lemma and the version of Gromov's local-to-global principle that we need.

\section{Proof of Theorem 1.4}

If $X$ is a 2-dimensional simplicial complex and $v \in F_{0}(X)$ is a vertex, define the link of $v$, denoted $\operatorname{lk}_{X}(v)$, to be the 1-dimensional simplicial complex (graph) with

$$
F_{0}\left(\operatorname{lk}_{X}(v)\right)=\left\{\{p\} \mid\{v, p\} \in F_{1}(X)\right\}
$$

and

$$
F_{1}\left(\operatorname{lk}_{X}(v)\right)=\left\{\{p, q\} \mid\{v, p, q\} \in F_{2}(X)\right\} .
$$

The key observation necessary to prove Theorem 1.4 is the following.

Lemma 2.1. For any $a, b, c \in[n]$ and simplicial complex $Y$ such that

(1) $\operatorname{lk}_{Y}(a) \cap \mathrm{lk}_{Y}(b)$ is connected and

(2) there exists $d \in[n]$ such that $\{a, b, d\} \in F_{2}(Y)$,

then the 3-cycle $\{\{a, b\},\{a, c\},\{b, c\}\}$ bounds an embedded disk in $Y$.

Proof. Since $\operatorname{lk}(a) \cap \mathrm{lk}(b)$ is connected, there exists a sequence $\left\{x_{i}\right\}_{1}^{k}$ such that $c=x_{1}, d=x_{k}$, and $\left\{x_{i}, x_{i+1}\right\} \in \operatorname{lk}(a) \cap \operatorname{lk}(b)$ for all $i<k$. The edge $\left\{x_{i}, x_{i+1}\right\} \in$ $\operatorname{lk}(a) \cap \operatorname{lk}(b)$ if and only if $\left\{\left\{a, x_{i}, x_{i+1}\right\},\left\{b, x_{i}, x_{i+1}\right\}\right\} \subseteq F_{2}(Y)$. So we see that $\{\{a, b\},\{a, c\},\{b, c\}\}$ bounds an embedded disk, as in Figure 1

Note that for each pair of vertices $a, b \in[n]$ the distribution of

$$
\operatorname{lk}_{Y}(a) \cap \mathrm{lk}_{Y}(b)
$$

is identical to the Erdős-Rényi random graph $G\left(n-2, p^{2}\right)$. To complete the proof of Theorem 1.4 we show that if $p$ is sufficiently large, then the hypotheses of Lemma 2.1 are a.a.s. satisfied for every distinct $a, b \in[n]$. This requires bounding the probability that $G(n, p)$ is not connected when $p$ is a bit larger than the threshold of $\log (n) / n$. We delegate the proof of Lemma 2.2 to Appendix 1 . 


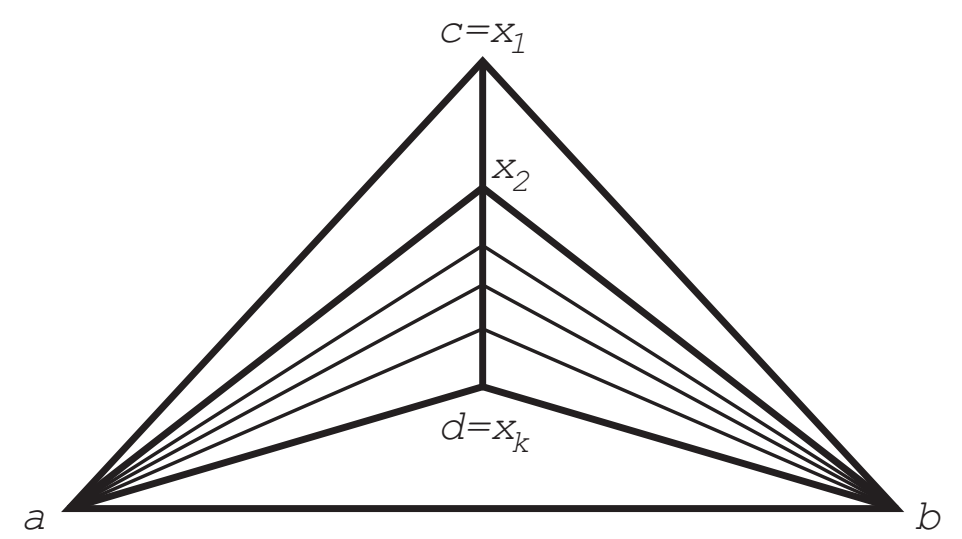

FigURE 1. Since $\operatorname{lk}(a) \cap \operatorname{lk}(b)$ is connected and edge $a b$ is contained in at least one face, the 3 -cycle $a b c$ bounds an embedded topological disk.

Lemma 2.2. Let $\omega(n) \rightarrow \infty$ as $n \rightarrow \infty$. If $p=\left(\frac{3 \log n+\omega(n)}{n}\right)^{1 / 2}$, then a.a.s.

(1) $\operatorname{lk}_{Y}(a) \cap \mathrm{lk}_{Y}(b)$ is connected and

(2) there exists $d \in[n]$ such that $\{a, b, d\} \in F_{2}(Y)$

for all distinct $\{a, b\} \subseteq[n]$ a.a.s.

Proof of Theorem 1.4. By Lemmas 2.1 and 2.2 we have that a.a.s. every 3 -cycle is contractible. That $Y$ is a.a.s. simply connected follows as $F_{1}(Y)$ is the complete graph and every $k$-cycle in the fundamental group is a product of 3 -cycles.

A more complicated version of this argument was used in 9 to prove the vanishing of the $k$ th homology $H_{k}$ for arbitrary $k$, for a different kind of random simplicial complex.

\section{Outline of Theorem 1.5}

3.1. Notation. For a 2-dimensional simplicial complex $X$ we write $F_{0}=F_{0}(X)$, $F_{1}=F_{1}(X)$ and $F_{2}=F_{2}(X)$ for the sets of vertices, edges and faces of $X$ and $f_{i}=\left|F_{i}\right|$ for the respective numbers. For an edge $e \in F_{1}(X)$ we write $f_{e}^{2}(X)=$ $\left|\left\{t \in F_{2}(X): e \subset \partial(t)\right\}\right|$ for the number of 2-faces containing $e$ in their boundaries.

Definition 3.1. We define $C_{r}$ to be the cycle of length $r$ with $F_{0}\left(C_{r}\right)=[r]=$ $\{1, \ldots, r\}([0]=\emptyset)$ and

$$
F_{1}\left(C_{r}\right)=\bigcup_{i=1}^{r-1}\{\{i, i+1\}\} \cup\{\{r, 1\}\} .
$$

Definition 3.2. Let $\gamma: C_{r} \rightarrow X$. We say that $\left(C_{r} \stackrel{b}{\rightarrow} D \stackrel{\pi}{\rightarrow} X\right)$ is a filling of $\gamma$ if $\gamma=\pi b$ and the mapping cylinder $\operatorname{Cyl}(b)$ of $b$ is a disk with boundary $C_{r} \times 0$.

Definition 3.3. Define the area of a curve $\gamma$ to be

$$
A(\gamma)=\min \left\{f_{2}(D) \mid(C \stackrel{b}{\rightarrow} D \stackrel{\pi}{\rightarrow} X) \text { is a filling of } \gamma\right\}
$$



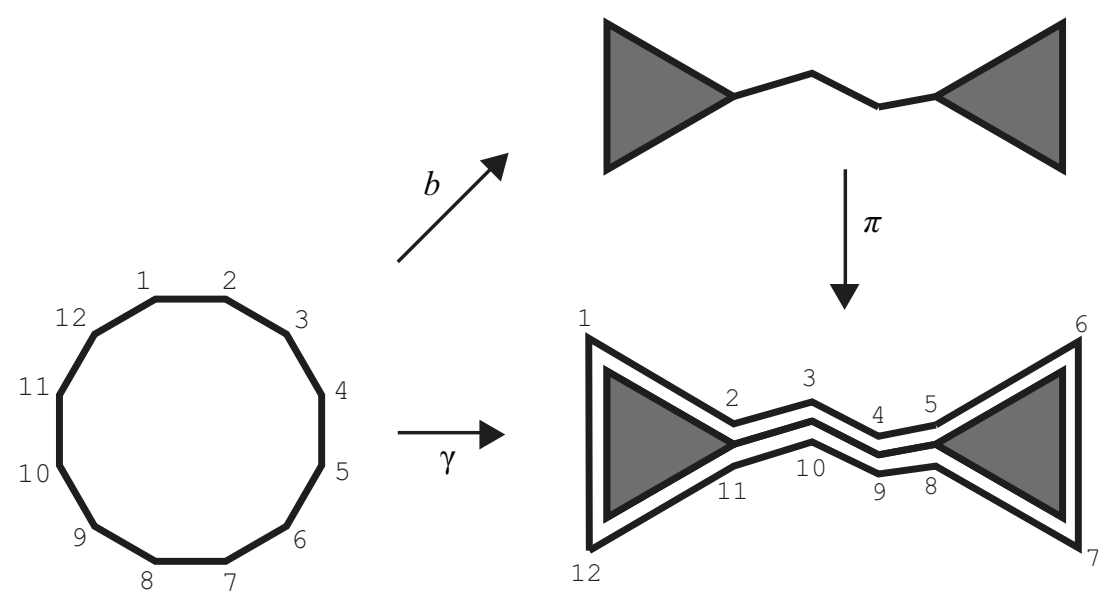

Figure 2. A minimal filling of a 12-cycle with $A(\gamma)=2$.

if $\gamma$ is contractible and $A(\gamma)=\infty$ if $\gamma$ is not contractible. We say that a filling $(C \stackrel{b}{\rightarrow} D \stackrel{\pi}{\rightarrow} X)$ of $\gamma$ is minimal if $A(\gamma)=f_{2}(D)$.

See Figure 2 for an example of a filling.

3.2. Sketch of proof of Theorem [1.5. Write $\operatorname{Id}_{[3]}:[3] \rightarrow[3]$ for the identity map. We show that for a typical $Y$ (with probability approaching 1 ) the cycle $\operatorname{Id}_{[3]}$ is not contractible and thus $Y$ is not simply connected. The main step is to prove a linear isoperimetric inequality. This means that there exists $\rho^{\prime}=\rho^{\prime}(\epsilon)$ such that for a typical $Y$ and for any $\gamma: C_{r} \rightarrow Y$, either $A(\gamma)=\infty$ or

$$
A(\gamma) \leq \rho^{\prime} r \text {. }
$$

Once we have a linear isoperimetric inequality for a typical $Y$, then we have

$$
\mathbf{P}\left(3 \rho^{\prime}<A\left(\operatorname{Id}_{[3]}\right)<\infty\right) \rightarrow 0 .
$$

Then we complete the proof by showing that

$$
\mathbf{P}\left(A\left(\operatorname{Id}_{[3]}\right) \leq 3 \rho^{\prime}\right) \rightarrow 0 .
$$

To carry out this program we introduce the following definitions. Throughout this section, $X$ is a 2-complex with vertex set $F_{0}(X)=[n]$.

Definition 3.4. We write

$$
e(X)=\min _{Z \subseteq X}\left(\frac{f_{0}(Z)}{f_{2}(Z)}\right) .
$$

More generally, if $[w] \subseteq F_{0}(X)$, then we write

$$
e_{w}(X)=\min _{\substack{Z \subset X \\[w] \subset F_{0}(Z)}}\left(\frac{f_{0}(Z)-w}{f_{2}(Z)}\right) .
$$

We say that $X$ is $\epsilon$-admissible if $e(X) \geq \frac{1}{2}+\epsilon$. For some $w \leq n$ we say that $X$ is $(\epsilon, w)$-admissible if $e_{w}(X) \geq \frac{1}{2}+\epsilon$. We say that a 2-complex $X$ is admissible $(w$ admissible) if there exists some $\epsilon>0$ such that $X$ is $\epsilon$-admissible $((\epsilon, w)$-admissible). 
We define things generally for convenience in notation, but in fact we will only ever use the case $w=3$.

The following lemma is the first step in showing the existence of a linear isoperimetric inequality.

Lemma 3.5. For every $\epsilon>0$ there exists $\rho$ such that for every $X$ with $e(X)>\frac{1}{2}+\epsilon$ and $\gamma: C_{r} \rightarrow X$, either $A(\gamma)=\infty$ or

$$
A(\gamma) \leq \rho r .
$$

The proof of Lemma 3.5 appears in Section 5 and requires the use of several other lemmas in between. The key to proving Lemma 3.5 is to analyze the topology of $\epsilon$-admissible complexes. In Lemma 4.1 we show that every $\epsilon$-admissible 2-complex is homotopy equivalent to a wedge product of circles, spheres and projective planes.

We cannot apply Lemma 3.5 directly to get a linear isoperimetric inequality for $Y$ because for a typical $Y$ we have that $f_{2}(Y)=O\left(n^{2}\right)$ (since we may assume $\epsilon<1 / 2)$ and $f_{0}(Y)=n$. Thus $e(Y)=O\left(\frac{1}{n}\right)$. Instead we analyze the subcomplexes $X \subset Y$ with $f_{2}(X)$ small. The next lemma tells us which small subcomplexes can be embedded in a typical $Y$.

Definition 3.6. For simplicial complexes $Z$ and $X$ with $F_{0}(Z) \cup F_{0}(X) \subset \mathbb{Z}^{+}$, and with $[w] \subseteq F_{0}(Z) \cap F_{0}(X)$, a $w$-inclusion $g$ of $Z$ into $X$ is an injective simplicial map $g: Z \rightarrow X$ such that $g(i)=i$ for all $1 \leq i \leq w$.

Definition 3.7. Let $X$ be a simplicial complex with $F_{0}(X)=[n]$ for some $n$. $X$ is $(\epsilon, m)$-sparse if for every 2-complex $Z$ with

(1) $f_{2}(Z) \leq m$ and

(2) $f_{0}(Z)<\left(\frac{1}{2}+\epsilon\right) f_{2}(Z)$

there is no embedding of $Z$ in $X$. $X$ is called $(\epsilon, m, 3)$-sparse if for every 2 -complex $Z$ with

(3) $[3] \subseteq F_{0}(Z)$,

(4) $f_{2}(Z) \leq m$ and

(5) $f_{0}(Z)-3<\left(\frac{1}{2}+\epsilon\right) f_{2}(Z)$

there is no 3-inclusion of $Z$ into $X$.

Lemma 3.8. For every $m \in \mathbb{Z}^{+}, \epsilon>0$, and $p=O\left(n^{-\frac{1}{2}-\epsilon}\right)$, we have that $Y \in$ $Y(n, p)$ is $(\epsilon, m, 3)$-sparse a.a.s.

Proof. For fixed $m$ and $r$ there are only finitely many complexes $Z$ with $f_{2}(Z)<m$. Thus to prove that $Y$ is $(\epsilon, m, 3)$-sparse a.a.s. we only need to prove that for any given complex $Z$ which does not satisfy conditions 3,4 and 5 that

$$
\mathbf{P}(Z \text { has a } 3 \text {-inclusion in } Y)=0 \text { a.a.s. }
$$

If $Z$ does not satisfy conditions 3,4 and 5 , then

$$
f_{0}(Z)-3<\left(\frac{1}{2}+\epsilon\right) f_{2}(Z)
$$


and hence

$$
\begin{aligned}
\mathbf{P}(Z \text { has a 3-inclusion into } Y) & \leq \mathbf{E}(\text { number of 3-inclusions of } Z \text { into } Y) \\
& \leq n^{f_{0}(Z)-3} p^{f_{2}(Z)} \\
& \leq n^{f_{0}(Z)-3} C^{f_{2}(Z)} n^{-\left(\frac{1}{2}+\epsilon\right) f_{2}(Z)} \\
& <C^{m} n^{-\alpha}
\end{aligned}
$$

for some $\alpha>0$.

We establish a linear isoperimetric inequality for $Y$ by combining Lemmas 3.5 and 3.8, together with Gromov's local-to-global principle. Similar results for groups appear in [6] and [13, but we require the result for 2-dimensional simplicial complexes, so we include a proof in Appendix 2 for the sake of completeness.

Theorem 3.9. If $X$ is a finite simplicial complex for which every $\gamma: C_{r} \rightarrow X$ satisfying $A(\gamma) \geq 44 \rho^{2}$ or $A(\gamma) \leq \frac{\rho}{44} r$ also has every $\gamma: C_{r} \rightarrow X$ satisfying $A(\gamma)=\infty$ or $A(\gamma) \leq \rho r$.

The local-to-global principle gives us the following.

Lemma 3.10. For every $\epsilon>0$ there exist $m$ and $\rho^{\prime}$ such that every $(\epsilon, m)$-sparse complex $X$ and every $\gamma: C_{r} \rightarrow X$ satisfies either $A(\gamma)=\infty$ or

$$
A(\gamma)<\rho^{\prime} r .
$$

Proof. Given $\epsilon>0$ choose $\rho$ as in Lemma 3.5 and then $K$ and $\rho^{\prime}$ as in Theorem 3.9. Thus by Lemma 3.5 the hypothesis of Theorem 3.9 is satisfied. Thus by Theorem 3.9. the lemma holds.

The same technology that we use to prove Lemma 3.5 can also be used to prove the following lemma.

Lemma 3.11. For every $X$ such that $[3] \subseteq F_{0}(X)$ with $e_{3}(X)>0$ the curve $I d_{[3]}$ is not contractible in $X$.

The proof of Lemma 3.11 appears in Section 5. Once we have established these lemmas we complete the proof by showing that in a typical complex that the curve $\mathrm{Id}_{[3]}$ is not contractible.

Lemma 3.12. For any $\epsilon>0$ there exists an $m$ such that for every 2-complex $X$ which is $(\epsilon, m, 3)$-sparse, the curve $I d_{[3]}$ is not contractible in $X$.

Proof. For $\epsilon>0$ choose $\rho$ as in Lemma 3.5 and then $K$ and $\rho^{\prime}$ as in Theorem 3.9, Then set $m=\max \left(K, 3 \rho^{\prime}\right)$. As $X$ is $(\epsilon, m, 3)$-sparse, every $Z \subset X$ with [3] $\in F_{0}(Z)$ and $f_{2}(Z) \leq 3 \rho^{\prime} \leq m$ satisfies $e_{3}(Z)>\epsilon$. Thus by Lemma 3.11 the curve $\operatorname{Id}_{[3]}$ is not contractible in $Z$. Thus $A\left(\operatorname{Id}_{[3]}\right)>3 \rho^{\prime}$.

By Lemma 3.8, $X$ is a.a.s. $(\epsilon, m, 3)$-sparse. Thus every $Z \subset X$ with $[3] \in F_{0}(Z)$ and $f_{2}(Z)<K \leq m$ satisfies $e_{3}(Z)>\epsilon$. Thus the hypotheses of Lemma 3.5] are satisfied for every such $Z$. Thus $X$ satisfies the hypotheses of Theorem 3.9 and $A\left(\operatorname{Id}_{[3]}\right)=\infty$ or $A\left(\operatorname{Id}_{[3]}\right)<3 \rho^{\prime}$. Thus $A\left(\operatorname{Id}_{[3]}\right)=\infty$ and $\operatorname{Id}_{[3]}$ is not contractible in $X$.

Proof of Theorem 1.5. That $\pi_{1}(Y)$ is nontrivial follows from Lemmas 3.8 and 3.12 . 
That it is hyperbolic follows from Lemmas 3.8 and 3.10, as follows. If there is a linear isoperimetric inequality on $Y$, then there is a linear isoperimetric inequality on $\pi_{1}(Y)$ as well; indeed it is well known that $\pi_{1}(Y)$ is quasi-isometric to $\widetilde{Y}$, the universal cover of $Y$. Groups which satisfy a linear isoperimetric inequality also satisfy a "thin triangles" condition and are Gromov hyperbolic [5].

\section{4. Номотору type of ADMissible 2-COMPLEXES}

The following lemma is a strengthening of Theorem 1.8 .

Lemma 4.1. If $X$ is an admissible, finite, 2-dimensional simplicial complex, then every connected component of $|X|$ has the homotopy type of a wedge of circles, spheres and real projective planes. Thus $\pi_{1}(X)$ is isomorphic to a free product of $\mathbb{Z} s$ and $\mathbb{Z} / 2 \mathbb{Z} s$ and it is a hyperbolic group.

Moreover there is a subcomplex $Z \subseteq X$ with $F_{1}(Z)=F_{1}(X)$ for which the inclusion induces an isomorphism of fundamental groups and $\chi\left(Z^{\prime}\right) \leq 1$ for any connected subcomplex $Z^{\prime} \subseteq Z$.

The proof of Lemma 4.1 requires several other intermediate results and appears at the end of this section.

4.1. Stratified complexes and webs. The proof of Lemma 4.1 is by induction. We assume by way of contradiction that there is a minimal counterexample and make reduction moves to find a smaller one. However, there is a fairly serious complication in that the reduction moves do not always leave us with a simplicial complex. For this reason we introduce the following more general complexes.

For a compact manifold with boundary $M$ we use the notation $\partial M$ for the boundary of $M$, and $M^{\circ}$ for the interior. (For a 0 -dimensional $M_{0}, \partial M_{0}=\emptyset$ and $\left.M_{0}^{\circ}=M_{0}.\right)$

Definition 4.2. A (2-dimensional) stratified complex $N$ consists of

(1) a topological space $N$ homeomorphic to the realization of a finite simplicial 2-complex,

(2) for each $i \in\{0,1,2\}$, a compact $i$-dimensional manifold with boundary $M_{i}$ (not necessarily connected), and

(3) immersions $\psi_{i}: M_{i} \rightarrow N$ such that the restrictions to interiors $\left.\psi_{i}\right|_{M_{i}}$ are embeddings, the images $\psi_{i}\left(M_{i}^{\circ}\right)$ partition $N$, and $\psi_{i}\left(\partial M_{i}\right) \subseteq \psi_{i-1}\left(M_{i-1}\right)$.

We call the connected components of $M_{i}$ the $i$-dimensional faces of $N$ and use the upper index to distinguish them. Then the set of $i$-dimensional faces of $N$ is denoted by $F_{i}(N)$ so that $M_{i}=\bigcup_{\phi \in F_{i}(N)} M_{i}^{\phi}$.

We refer the reader to Figure 3 for an example of a stratified complex. We note that the structure is not quite the same as a CW-complex, for example, since the cells need not be topological disks. In this example, $M_{2}$ is the disjoint union of two disks and a cylinder, $M_{1}$ is the disjoint union of a line segment and a circle, and $M_{0}$ is a point. If one of the disks in $M_{2}$ were replaced with a cross-cap, $N$ would be homeomorphic to a projective plane. 


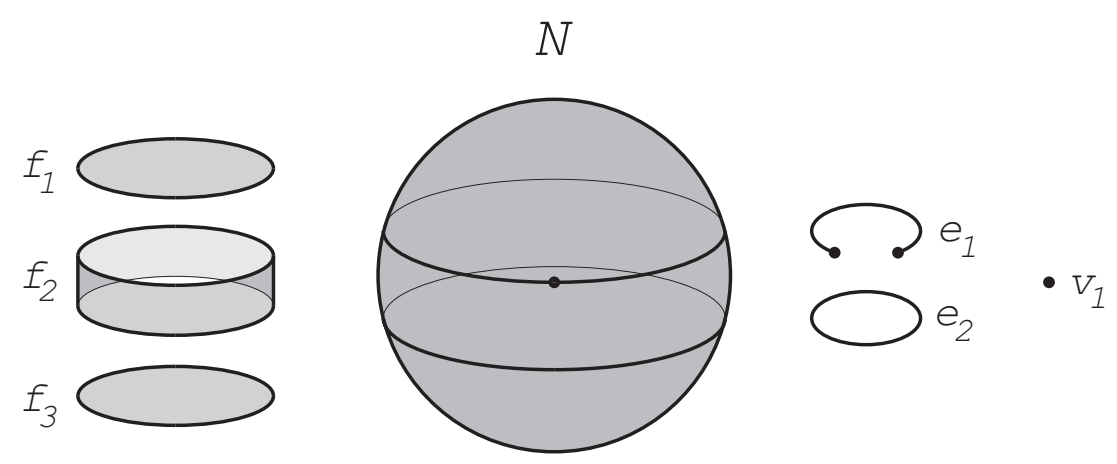

Figure 3. A stratified complex $N$, homeomorphic to a sphere. In this example, $F_{0}(N)=1, F_{1}(N)=2$, and $F_{2}(N)=3$, and we also have, for example, that $f_{1}^{v}=2 . f_{2}^{e_{1}}=2, f_{2}^{v}=2$.

Definition 4.3. If $N$ is a stratified complex, $i<i^{\prime}, u \in F_{i}(N)$ and $u^{\prime} \in F_{i^{\prime}}(N)$, then write:

(1) $f_{i}(N)=\left|F_{i}(N)\right|$,

(2) $f_{u^{\prime}}^{u}(N)=\left|\psi_{i}^{-1}(x) \cap M_{i^{\prime}}^{u^{\prime}}\right|$ for any $x \in \psi_{i}\left(M_{i}^{\circ u}\right)$,

(3) $f_{u^{\prime}}^{i}(N)=\sum_{u \in F_{i}(N)} f_{u^{\prime}}^{u}(W)$ and

(4) $f_{i^{\prime}}^{u}(N)=\sum_{u^{\prime} \in F_{i^{\prime}}(N)} f_{u^{\prime}}^{u}(W)$.

For every stratified complex $N$ and $e \in F_{1}(N), M_{1}^{e}$ is homeomorphic to either an interval or a circle. At times we need to distinguish these cases, so we introduce the following additional notation.

Definition 4.4. We write

(1) $F_{1, c}(N)=\left\{e \in F_{1}(N) \mid M_{1}^{e} \cong S^{1}\right\} \subseteq F_{1}(N)$,

(2) $f_{1, c}(N)=\left|F_{1, c}(N)\right|$ and

(3) for a vertex $u^{\prime} \in F_{0}(N)$ write $f_{u^{\prime}}^{1, c}=\sum_{u \in F_{1, c}(N)} f_{u^{\prime}}^{u}(W) \ldots$

Definition 4.5. A web $W$ is a stratified complex with an atom-free measure $\mu$ on $M_{1}$ which pulls back via $\psi$ to a measure (also $\mu$ ) on $\partial M_{2}$ with $\mu\left(M_{1}^{e}\right) \in \mathbb{N}$ for every $e \in F_{1}(W)$.

A subweb $W^{\prime}$ of a web $W$ is uniquely specified by subsets $F_{i}\left(W^{\prime}\right) \subseteq F_{i}(W)$. If $W$ is a web and $v \in F_{0}(W)$ is a vertex, then $\operatorname{lk}_{W}(v)$ is the link of $v$ in $W$ which is again a stratified complex, with $f_{i}\left(\mathrm{lk}_{W}(v)\right)=f_{i+1}^{v}(W)$.

Definition 4.6. If $X$ is a finite simplicial complex, then $W=W(X)$ is the associated web with $|X|=|W|, F_{i}(W)=F_{i}(X), M_{i}(W)=F_{i}(X) \times \Delta_{i}$ a disjoint union of standard simplices, $\psi$ restricted to each face is an embedding and every edge $e \in F_{1}$ has length one $\left(\mu M_{1}^{e}=1\right)$. Thus we can consider simplicial complexes as special cases of webs. 
Two very useful functions from webs to integers are the Euler characteristic $\chi(W)=\chi(|W|)$ and the length

$$
L(W)=2 \mu\left(M_{1}(W)\right)-\mu\left(\partial M_{2}(W)\right) .
$$

Definition 4.7. We say that a nonempty web $W$ is admissible if every nonempty subweb $W^{\prime}$ satisfies

$$
(2 \chi+L)\left(W^{\prime}\right)>0 .
$$

Definition 4.8. Other useful functions from webs to integers include

(1) $d(W)=\max \left\{i \mid f_{i}(W)>0\right\}$ (dimension),

(2) $\delta(W)=\min \left\{f_{i}^{u} \mid u \in F_{i-1}(W), 0<i \leq d(W)\right\}$ (minimum degree) and

(3) $g(W)=\min \left\{\mu(S) \mid f: S \rightarrow \psi M_{1}\right.$ is an isometric embedding of a circle $\}$ (girth).

Definition 4.9. A web $W$ is a refinement of another web $W^{\prime}$ if there is a homeomorphism $r:|W| \rightarrow\left|W^{\prime}\right|$ and for each $u \in F_{i}\left(W^{\prime}\right)$ there exists a subweb $W^{u}$ of $W$ with the restriction of $r$ to $\left|W^{u}\right|$ a homeomorphism onto $\psi\left(M_{i}^{u}\right)$. If $i=1$ there is a measure on $\psi\left(M_{1}^{u}\right)$ pulling back to $\mu$ and $\mu^{\prime}$.

Up to isomorphism, a refinement depends only on the subwebs $\left\{\left\{W^{u}\right\}_{u \in F_{i}\left(W^{\prime}\right)}\right\}_{i}$.

Lemma 4.10. If $X$ is a simplicial 2-complex, $W(X)$ is the associated web and $W(X)$ is a nontrivial refinement of $W^{\prime}$, then

(1) $g(W(X)) \geq 3$,

(2) if $X$ is admissible, then so is $W(X)$,

(3) $\chi\left(M_{2}\left(W^{\prime}\right)\right)=\sum_{t \in F_{2}\left(W^{\prime}\right)} \chi\left(M_{2}^{t}\right)$,

(4) $\chi\left(M_{0}\left(W^{\prime}\right)\right)=f_{0}\left(W^{\prime}\right)$,

(5) $\left|W^{\prime}\right| \cong|W|$,

(6) $d\left(W^{\prime}\right)=d(W)$

(7) $g\left(W^{\prime}\right)=g(W)$,

(8) $\delta(W)=\min \left\{\delta\left(W^{\prime}\right), 2\right\}$ and

(9) if $W$ is admissible, then so is $W^{\prime}$.

Proof. These all follow directly from the definitions.

Lemma 4.11. If $W$ is a 2-dimensional stratified complex so that no vertex link decomposes as a (nontrivial) wedge sum with a circle as one of the summands, then there is a unique stratified complex $C W$ such that

(1) $W$ is a refinement of $C W$ and

(2) $\delta(C W) \neq 2$.

Proof. The construction of $C W$ follows. Uniqueness is clear.

Set $M_{0, s}(W)=\left\{v \in M_{0}(W)||\left\{e \in F_{1}^{v} \mid f_{e}^{2} \neq 2\right\} \mid=s\right\}$.

Set $M_{0}(C W)=\bigcup_{s \notin\{0,2\}} M_{0, s}(W)$.

Set

$$
M_{1}(C W)=\left(\bigcup_{e \in F_{1}(W), f_{e}^{2} \neq 2} M_{1}^{e} \cup M_{0,2}(W)\right) / \sim,
$$

where $a \sim v$ if $a \in M_{1}(W), v \in M_{0,2}(W)$ and $\psi(a)=\psi(v)$.

Set

$$
M_{2}(C W)=M_{2}(W) \bigcup_{e \in F_{1}(W), f_{e}^{2}=2} M_{1}^{e} \cup M_{0,0}(W) / \sim,
$$


where $a \sim b$ if $\psi(a)=\psi(b)$ and either $a \in M_{1}(W)$ and $b \in M_{0}(W)$ or $a \in M_{2}^{t}$ and $b \in M_{1}^{e}$ and there is an inclusion of $M_{1}^{e}$ into $M_{2}^{t}$ commuting with $\psi$.

The map $\psi(C W): M(C W) \rightarrow|C W|=|W|$ is then inherited from $\psi$ for $W$.

It is now straightforward to check that each $M_{i}(C W)$ is a manifold with boundary, with interior points precisely the equivalence classes of points in the interior of some $M_{j}(W)$.

The other properties are straightforward to check.

Definition 4.12. A graph is 2-connected if it has at least three vertices and is connected after deleting any vertex.

Lemma 4.13. If $W$ is a web with all vertex links 2-connected, then the hypotheses of Lemma 4.11 hold.

Proof. The hypothesis of Lemma 4.11 is that no vertex link of $W$ decomposes as a (nontrivial) wedge sum with a circle as one of the summands. If some vertex link $\mathrm{lk}(v)$ did have such a decomposition, then by definition of wedge sum there would be a cut vertex in $l \mathrm{k}(v)$, contradicting the assumption that $\mathrm{lk}(v)$ is 2 -connected.

Now we introduce a collapsing construction.

Definition 4.14. If $A \subseteq W$ is a subcomplex of a 2 -dimensional stratified complex $W$, then write $K=K_{A}(W)$ for the maximal subcomplex of $W$ for which every edge $e \in F_{1}(K)$ either has $f_{e}^{2}(K) \geq 2$ or $f_{e}^{2}(K) \geq 1$ and $e \in F_{1}(A)$.

This collapsing construction is useful in this section with $A=\emptyset$ and again in the next section with more general $A$.

Lemma 4.15. For any web $W$ and $A \subset W$ each connected component of $|W|$ has the homotopy type of a wedge of components of $\left|K_{A}(W)\right|$ and circles. Also for any $W, \delta\left(K_{\emptyset}(W)\right) \geq 2$.

Proof. $K_{A}(W)$ is obtained from $W$ by a sequence of collapses of cells to wedges of circles (removing an edge in exactly one face) which induce homotopy equivalences and deletions of edges not contained in any face. The second statement follows straight from the definition.

Lemma 4.16. If $W$ is an admissible 2-dimensional web with $g(W) \geq 3$, then every connected component of $|W|$ has the homotopy type of a wedge of circles, spheres and projective planes.

Definition 4.17. Now we put a partial order on the webs. For $(i, j),\left(i^{\prime}, j^{\prime}\right) \in \mathbb{Z}^{2}$ we say that $(i, j)$ is before $\left(i^{\prime}, j^{\prime}\right)$ in the lexicographic order if either $i>i^{\prime}$ or $i=i^{\prime}$ and $j<j^{\prime}$. For any web $W$ let

$$
\left\{f_{i, j}(W)=\left|\left\{u \in F_{i}(W) \mid f_{u}^{i+1}=j\right\}\right|\right\}_{i, j} .
$$

Then to compare two webs $W$ and $W^{\prime}$ let $(i, j)$ be the first pair (in the lexicographic order) such that

$$
f_{i, j}(W) \neq f_{i, j}\left(W^{\prime}\right) .
$$

Then we say that $W$ is smaller than $W^{\prime}$ if $f_{i, j}(W)<f_{i, j}\left(W^{\prime}\right)$.

The proof of Lemma 4.16 requires a few intermediate lemmas, and is by induction with respect to the partial order we just defined. Whenever we refer to a minimal counterexample it is minimal with respect to this partial order. 
Lemma 4.18. If $W$ is a minimal counterexample to Lemma 4.16, then $\delta(W) \geq 3$.

Proof. We begin by showing that for a minimal counterexample $W$ that $W=$ $K_{\emptyset}(W)$ and that every vertex link of $W$ is two-connected (as a graph). Note that $K_{\emptyset}(W)$ is admissible, no larger than $W$ and has $W$ homotopy equivalent to the wedge sum of components of $K_{\emptyset}(W)$ and some circles. Thus $W=K_{\emptyset}(W)$ by minimality, and by Lemma 4.15 $\delta(W) \geq 2$.

Next we note that if $W$ has a vertex link which is not 2-connected, then splitting a vertex into two along a cut point gives a complex with smaller $\left\{f_{1, j}\right\}$ which is still admissible and homotopy equivalent to $W$. Splitting one between connected components gives a complex with smaller $\left\{f_{0, j}\right\}$ which is still admissible and $W$ is homotopy equivalent to the wedge of its connected components and some circles. Thus by minimality all vertex links of $W$ are 2 -connected and by Lemma 4.13 the hypotheses of Lemma 4.11 are satisfied. Thus $\delta(C W) \neq 2$.

Note that by Lemma 4.11 the web $C W$ exists, is no larger than $W$ and is homotopy equivalent to $W$. Thus as $W$ is minimal, $W=C W$. Thus we have $\delta(W)=\delta(C W) \neq 2$ and above we had that $\delta(W) \geq 2$. Combining these we get $\delta(W) \geq 3$.

Definition 4.19. A 2-face $t$ is digon if $t \in F_{2}(W)$ and there exists $e, f \in F_{0}(W)$ with $F_{t}^{1}=\{e, f\}$.

Lemma 4.20. If $W$ is a minimal counterexample to Lemma 4.16, then $W$ has no 2 -face that is a digon.

Proof. Note that if $t \in F_{2}(W)$, then $f_{t}^{1}>0$. If not, then we would have that $M_{2}^{t}=|W|$ is a connected 2-manifold and, as $W$ is admissible, that $\chi\left(M_{2}^{t}\right)>0$. Thus $W$ would be a sphere or projective plane and would not be a counterexample.

Note that if any 2-face is a digon, then construct $W^{\prime}$ by choosing a homeomorphism $\tau: M_{1}^{e} \rightarrow M_{1}^{f}$ compatible with $\psi$ on the boundaries and setting $M_{0}\left(W^{\prime}\right)=M_{0}(W), M_{1}\left(W^{\prime}\right)=M_{1}(W) /\{a \sim \tau(a)\}$ and $M_{2}\left(W^{\prime}\right)=M_{2}(W) / \sim$, where any two points of any folded interval are equivalent under $\sim$. An interval $I \subseteq \partial M_{2}(W)$ is folded if it has a midpoint $p \in I$ with $\left.\psi\right|_{I-\{p\}}$ two to one. Note that the homotopy type of $|W|$ is the wedge sum of all but one of the components of $\left|W^{\prime}\right|$ and some circles. Take $W^{\prime \prime}$ to be a connected component of $W^{\prime}$ which is not a wedge of circles, spheres and projective planes. $W^{\prime \prime}$ clearly has fewer faces than $W$ and is still admissible. Thus by minimality, $W$ has no digons.

Definition 4.21. Define $\bar{\mu}$ to be the measure with $\bar{\mu}\left(M_{1}^{e}\right)=1$ if $e \in F_{1}(W) \backslash$ $F_{1, c}(W)$ and $\bar{\mu}\left(M_{1}^{e}\right)=3$ if $e \in F_{1, c}(W)$.

Lemma 4.22. If $W$ is a minimal counterexample to Lemma 4.16, then either

(1) there exists $u \in F_{0}(W)$ such that

$$
\sum_{t \in F_{2}(W)}\left(f_{u}^{t} \frac{2 \chi\left(M_{2}^{t}\right)}{\bar{\mu}\left(\partial M_{2}^{t}\right)}-1\right)>-2
$$

or

(2) there exists $u \in F_{1, c}(W)$ such that

$$
\sum_{t \in F_{2}(W)}\left(f_{u}^{t} \frac{2 \chi\left(M_{2}^{t}\right)}{\bar{\mu}\left(\partial M_{2}^{t}\right)}-1\right)>-2 .
$$


Proof. For every $t \in F_{2}(W)$,

$$
1=\frac{f_{0}^{t}+3 f_{1, c}^{t}}{\bar{\mu}\left(\partial M_{2}^{t}\right)}
$$

and

$$
\chi(M)=\chi\left(M_{0}\right)-\chi\left(M_{1}\right)+\chi\left(M_{2}\right) .
$$

Let

$$
\bar{L}(N)=2 \bar{\mu}\left(M_{1}(N)\right)-\bar{\mu}\left(\partial M_{2}(N)\right) .
$$

Since $g(W) \geq 3$ we have $\mu \geq \bar{\mu}$. Since $\delta(W) \geq 3$ and

$$
L=\sum_{e \in F_{1}(W)}\left(2-f_{e}^{2}\right) \mu\left(M_{1}^{e}\right)
$$

we have

$$
L(W) \leq \bar{L}(W) .
$$

Finally we note that the definition of $\bar{\mu}$ gives us that

$$
(2 \bar{\mu}-2 \chi)\left(M_{1}(W)\right)=6 f_{1, c}(W)
$$

and

$$
\bar{\mu}\left(\partial M_{2}(W)\right)=\sum_{t \in F_{2}(W)} f_{0}^{t}+3 f_{1, c}^{t} .
$$

Note that

$$
\begin{aligned}
& 0<(2 \chi+L)(W) \\
& \leq(2 \chi+\bar{L})(W) \\
&= 2 \chi\left(M_{0}(W)\right)+(2 \bar{\mu}-2 \chi)\left(M_{1}(W)\right)+\left(-\bar{\mu}\left(\partial M_{2}(W)\right)+2 \chi\left(M_{2}(W)\right)\right) \text { by (77) } \\
&\text { by (15) and (6) }) \\
&= 2 f_{0}(W)+6 f_{1, c}(W)-\sum_{t \in F_{2}(W)}\left(f_{0}^{t}+3 f_{1, c}^{t}\right)+\sum_{t \in F_{2}(W)} \frac{f_{0}^{t}+3 f_{1, c}^{t}}{\bar{\mu}\left(\partial M_{2}^{t}\right)}(2 \chi)\left(M_{2}^{t}\right) \\
&(10)= \sum_{u \in F_{0}(W)}\left[2+\sum_{t \in F_{2}(W)}\left(f_{u}^{t} \frac{2 \chi\left(M_{2}^{t}\right)}{\bar{\mu}\left(\partial M_{2}^{t}\right)}-1\right)\right] \\
&+\sum_{u \in F_{1, c}(W)}\left[3\left[2+\sum_{t \in F_{2}(W)}\left(f_{u}^{t} \frac{2 \chi\left(M_{2}^{t}\right)}{\bar{\mu}\left(\partial M_{2}^{t}\right)}-1\right)\right]\right. \text { and Lemma 4.10 }
\end{aligned}
$$

Since the sum is positive at least one of the summands in (10) or (11) must be positive. Rearranging the summands completes the proof. 
Lemma 4.23. If there exists $u \in F_{1, c}(W)$ with

$$
\sum_{t \in F_{2}(W)}\left(f_{u}^{t} \frac{2 \chi\left(M_{2}^{t}\right)}{\bar{\mu}\left(\partial M_{2}^{t}\right)}-1\right)>-2,
$$

then $W$ is not a minimal counterexample to Lemma 4.16.

Proof. Fix such a $u \in F_{1, c}(W)$. For a face $t \in F_{2}(W)$ to contribute more than $-f_{u}^{t}$ to the sum in (10) or (11) we must have $\chi\left(M_{2}^{t}\right)>0$, which implies that $M_{2}^{t}$ is a disk. Since by Lemma 4.20, $W$ has no digons, every face contributes at least $-\frac{1}{3} f_{u}^{t}$ with equality only if $M_{2}^{t}$ is a (an embedded) triangle (or $f_{u}^{t}=0$ ).

If $u \in F_{1, c}(W)$, then by Lemma 4.18 there are at least $f_{u}^{2} \geq \delta(W) \geq 3$ (weighted) terms, including either two embedded disks $t, t^{\prime} \in F_{u}^{2}$ with $\partial M_{2}^{t}=\partial M_{2}^{t^{\prime}}=\psi\left(M_{1}^{u}\right)$, or the entire complex is the union of a projective plane with a disk along an embedded circle. In the former case, deleting $t$ gives $W^{\prime}$, which is clearly a smaller admissible counterexample, contradicting minimality. In the latter case the entire complex has the homotopy type of a sphere and thus it is not a counterexample.

Lemma 4.24. If there exists $u \in F_{0}(W)$ with

$$
\sum_{t \in F_{2}(W)}\left(f_{u}^{t} \frac{2 \chi\left(M_{2}^{t}\right)}{\bar{\mu}\left(\partial M_{2}^{t}\right)}-1\right)>-2
$$

then $W$ is not a minimal counterexample to Lemma 4.16 .

Proof. Fix such a $u \in F_{0}(W)$. Then there are at least $f_{u}^{2} \geq \frac{9}{2}$ (weighted) terms, including two embedded disks $t, t^{\prime} \in F_{u}^{2}$ with $\mu(\partial t)=\mu\left(\partial t^{\prime}\right)=3$ and $\mu\left(\partial t \cap \partial t^{\prime}\right) \in$ $\{2,3\}$. One sees this by explicitly enumerating all ways to get a positive term with at least 3 vertices and 5 edges in the link of $u$. It turns out that the link must be a triangle with two edges doubled and at least 4 of the edges must come from triangles and hence all 5 must be embedded. Let $t$ and $t^{\prime}$ be two triangles forming a double edge in the link of $u$. If $\partial t=\partial t^{\prime}$, deleting $t$ gives a smaller counterexample contradicting minimality as above.

If $\partial t \neq \partial t^{\prime}$, then a web $W^{\prime}$ with the same homotopy type as $W$ and one fewer 2face exists. $W^{\prime}$ is obtained by deleting $t$ and identifying the two edges in $\left(\partial t \cup \partial t^{\prime}\right) \backslash$ $\left(\partial t \cap \partial t^{\prime}\right)$. It remains to check that $W^{\prime}$ is admissible, contradicting the minimality of $W$ and completing the proof. Checking admissibility is straightforward.

Proof of Lemma 4.16. Assume that $W$ is a minimal counterexample. Lemmas 4.22, 4.23 and 4.24 form a contradiction.

Proof of Lemma 4.1. We note that by Lemma 4.10 for any simplicial complex $X$ that $g(W(X)) \geq 3$. Thus Lemma 4.16 applies to all admissible complexes. This tells us that for every admissible complex $X$, the homotopy type of $X$ is a wedge product of circles, spheres and projective planes. This proves the first claim in the lemma, which is the same as Theorem 1.8 .

To prove the second claim choose $j: Z \rightarrow X$ to be a minimal subcomplex such that $\pi_{1}(j)$ is an isomorphism and $f_{1}(Z)=f_{1}(X)$ (e.g. $Z=X$ ). If $Z \simeq S^{2} \vee Z^{\prime}$, then choose a simplicial map $f: S \rightarrow Z$ with $|S| \cong S^{2}$ and $H_{2}(f ; \mathbb{Z} / 2 \mathbb{Z}) \neq 0$ and a 2-face $t$ of $Z$ with $\left|f^{-1}(t)\right|$ odd. Fix a presentation of

$$
\pi_{1}(Z \backslash t)=\left\langle a_{1}, \ldots, a_{s}, b_{1}, \ldots, b_{t} \mid b_{i}^{2}=1 \forall i=1, \ldots, t\right\rangle
$$


and express some element of $\pi_{1}(Z \backslash t)$ represented by the boundary of $t$ as a cyclically reduced word $[\partial t]=w_{1} w_{2} \ldots w_{v}$. The restriction $\left.f\right|_{S \backslash f^{-1}(t)}$ shows that $[\partial t]^{r}=1$ for some odd $r$, so $w_{1}=w_{v}^{-1}$ and hence $v \leq 1$ and $[\partial t]=1$. Note that

$$
\pi_{1}(i): \pi_{1}(Z \backslash t) \rightarrow \pi_{1}(Z \backslash t) /\langle[\partial t]\rangle=\pi_{1}(Z)
$$

is the quotient map (where $\langle\ldots\rangle$ is the normal closure) and hence an isomorphism, contradicting the minimality of $Z$.

\section{ISOPERIMETRIC INEQUALITIES}

Classifying the homotopy type of admissible complexes $X$ is a major step towards establishing a linear isoperimetric inequality for $Y$. However we also need a bound on the number of faces in the spheres and projective planes. (A family of spheres with an increasing number of vertices need not satisfy any one linear isoperimetric inequality.)

To get this bound we now recall the function $L$ (previously defined for webs) which generalizes the length of the boundary of a disk:

$$
L(X)=2 f_{1}(X)-3 f_{2}(X)=\sum_{e \in F_{1}(X)}\left(2-f_{e}^{2}\right) .
$$

Lemma 5.1. If $X$ is an $(\epsilon, w)$-admissible 2-complex, then

$$
f_{2}(X) \leq \frac{2 \chi(X)-2 w+L(X)}{2 e_{w}(X)-1} .
$$

Proof. By the definitions of $\chi, e_{w}$ and $L$ we get

$$
\left[\begin{array}{rrr}
1 & -1 & 1 \\
-1 & 0 & e_{w}(X) \\
0 & 2 & -3
\end{array}\right]\left[\begin{array}{l}
f_{0}(X) \\
f_{1}(X) \\
f_{2}(X)
\end{array}\right] \leq\left[\begin{array}{c}
\chi(X) \\
-w \\
L(X)
\end{array}\right]
$$

Multiplying both sides on the left by $[2,2,1]$ gives the desired result.

Lemma 5.2. There exists $\beta=\beta(\epsilon)>1$ with the following property. Let $X$ be an $\epsilon$-admissible connected 2-complex with $L(X) \leq 0$ and $\chi(X) \leq 1$. For any $r \in \mathbb{N}$ and any $\gamma: C_{r} \rightarrow X$ which is null-homotopic in $X$ we have

$$
A(\gamma)<\beta r .
$$

Proof. We first consider the case when $k=1$. As $L(X) \leq 0$ and $\chi(X) \leq 1$, Lemma 5.1 implies that $f_{2}(X) \leq 1 / \epsilon$. Since $L(X) \leq 0$ we also have an upper bound on $f_{1}(X)$, and since $X$ is connected, this gives an upper bound on $f_{0}(X)$. So there are only a finite number of $X$ that satisfy the hypotheses of the lemma. By Lemma 4.1 we have that $\pi_{1}(X)$ is a free product of $\mathbb{Z}$ and $\mathbb{Z} / 2 \mathbb{Z}$ terms. A free product of hyperbolic groups is hyperbolic, so $\pi_{1}(X)$ is hyperbolic. (Here we mean "word hyperbolic" in the sense of Gromov [5].) Hence we have a linear isoperimetric inequality on $\pi_{1}(X)$ with respect to any presentation of the group.

Let $T$ denote any spanning tree of $X$, and let $X / T$ denote the quotient of $X$ with all the points of $T$ identified to a single point. We can endow $X / T$ with the structure of a CW-complex with one vertex, and $X / T$ is easily seen to be a presentation complex for $\pi_{1}(X)$. 
Let $\pi: X \rightarrow X / T$ be the natural projection map. By assumption, $\gamma\left(C_{r}\right)$ is null-homotopic in $X$, so $b\left(C_{r}\right)=\pi^{-1} \gamma\left(C_{r}\right)$ is a trivial word in $\pi_{1}(X)$. Hence there is some constant $\beta_{X}$ such that

$$
A(\pi \gamma)<\beta_{X} L\left(b\left(C_{r}\right)\right)
$$

We also have that

$$
L\left(b\left(C_{r}\right)\right) \leq r
$$

since some edges may get contracted in the projection, but the cycle cannot get longer.

Thus there is a $\beta_{X}$ such that

$$
A(\gamma)<\beta_{X} r
$$

for all null-homotopic curves $\gamma$ in $X$. As there are only finitely many such $X$ we can set $\beta=\max _{X} \beta_{X}$, and we have that for all $\gamma$ and $X$,

$$
A(\gamma)<\beta r
$$

as desired.

\subsection{Proofs of Lemmas 3.5 and 3.11.}

Definition 5.3. A simplicial map $f: X \rightarrow Y$ is a $d$-immersion if the restriction to the star of any $d$-face is an embedding.

Lemma 5.4. If $\left(C_{r} \stackrel{b}{\rightarrow} D \stackrel{\pi}{\rightarrow} X\right)$ is a minimal filling and $\gamma=\pi b$ is a 0 -immersion, then $\pi$ is a 1-immersion.

Proof. See the appendix for the notation. Assume not and choose vertices $u \neq v$ of $D$ with $\pi(u)=\pi(v)$ and $B(1, u) \cap B(1, v)$ containing at least one edge. Take $x$ and $y$ to be the two points of $B(1, u) \cap B(1, v)$ in the closure of $D-\langle B(1,\{u, v\})\rangle_{\operatorname{Im}(b)}$. Consider the length-4 loop $\delta: C_{4} \rightarrow D$ with vertices $v, x, u$ and $y$. Construct a smaller filling

$$
\left(C_{r} \stackrel{b}{\rightarrow} D^{\prime} \stackrel{\left.\pi\right|_{D^{\prime}}}{\longrightarrow}, X\right)
$$

of $\gamma$, where

$$
D^{\prime}=\left(\left(D-\left\langle\delta C_{4}\right\rangle_{\operatorname{Im}(b)}\right) \cup \delta C_{4}\right) /(u \sim v) .
$$

Let $Z$ be any 2-complex, $r \in \mathbb{N}, \gamma$ be a 0 -immersion $\gamma: C_{r} \rightarrow Z$ and $\left(C_{r} \stackrel{b}{\rightarrow} D \stackrel{\pi}{\rightarrow}\right.$ $X$ ) be a minimal filling of $\gamma$. To show that $r>\rho f_{2}(D)$ we will define a subcomplex $D_{L \leq 0} \subset D$. Then we break the proof up into two cases. Lemma 5.9 will cover the case that $f_{2}\left(D \backslash D_{L \leq 0}\right) / f_{2}(D)$ is bounded away from 0 . Lemma 5.10 will cover the case that $f_{2}\left(D_{L \leq 0}\right) / f_{2}(D)$ is close to 1 .

If $A \subset D$ we define $D \backslash A \subseteq D$ to be the pure 2-dimensional subcomplex with $F_{2}(D \backslash A)=F_{2}(D) \backslash F_{2}(A)$. We now define $D_{L \leq 0}$.

Definition 5.5. Define the pure 2-complexes $Z_{i} \subseteq Z$ with

$$
F_{2}\left(Z_{i}\right)=\left\{z \in F_{2}\left(Z^{\prime}\right):\left|\pi^{-1}(z)\right| \geq i\right\} .
$$

For each $i$ enumerate the connected components of $Z_{i}$ by $\left\{Z_{i, j}\right\}_{j}$. Let $Q$ be the union of the index sets of the connected components of the $Z_{i}$. Define

$$
Q_{L \leq 0}=\left\{(i, j) \in Q: L\left(Z_{i, j}\right) \leq 0\right\}
$$


and

$$
\bar{Q}_{L>0}=\left\{(i, j) \in Q: \forall\left(i^{\prime}, j^{\prime}\right) \text { with } Z_{i, j} \subset Z_{i^{\prime}, j^{\prime}} \text { we have } L\left(Z_{i^{\prime}, j^{\prime}}\right)>0\right\}
$$

Then define

$$
Z_{L \leq 0}=\bigcup_{(i, j) \in Q_{L \leq 0}} Z_{i, j} \subseteq Z
$$

and $D_{L \leq 0} \subseteq D^{\prime}$ by

$$
F_{2}\left(D_{L \leq 0}\right)=\left\{d \in F_{2}(D) \mid \pi(d) \in Z_{L \leq 0}\right\} .
$$

Lemma 5.6. For any $Z$ and 0 -immersion $\gamma: C_{r} \rightarrow Z$, any minimal filling $\left(C_{r} \stackrel{b}{\rightarrow}\right.$ $D \stackrel{\pi}{\rightarrow} X)$ of $\gamma$ satisfies

$$
r \geq \sum_{(i, j) \in \bar{Q}_{L>0}} L\left(Z_{i, j}\right)
$$

Note that the lemma also holds with $\bar{Q}_{L>0}$ replaced by any order ideal in $Q$.

Proof. For every edge $e \in F_{1}(Z)$ and $Q^{\prime} \subset Q$ define

$$
|e|_{\infty}^{Q^{\prime}}=\max _{f \in F_{2}(Z): e \in \partial f}\left|\left\{\left(i^{\prime}, j^{\prime}\right) \in Q^{\prime}: f \in F_{2}\left(Z_{i^{\prime}, j^{\prime}}\right)\right\}\right|
$$

and

$$
|e|_{1}^{Q^{\prime}}=\sum_{f \in F_{2}(Z): e \in \partial f}\left|\left\{\left(i^{\prime}, j^{\prime}\right) \in Q^{\prime}: f \in F_{2}\left(Z_{i^{\prime}, j^{\prime}}\right)\right\}\right| .
$$

By Lemma 5.4 the filling $(C \stackrel{b}{\rightarrow} D \stackrel{\pi}{\rightarrow} X)$ is a 1-immersion. Thus for any $e$,

$$
f_{1}\left(\pi^{-1} e \cap \partial D\right) \geq \max \left(0,\left(2|e|_{\infty}^{Q}-|e|_{1}^{Q}\right)\right)
$$

For any $e$ if there exists $(i, j) \notin \bar{Q}_{L>0}$ and $g, h \in F_{2}\left(Z_{i, j}\right)$ such that $e \in \partial(g \cap h)$, then the maximum in the definition of $|e|_{\infty}^{\bar{Q}_{L>0}}$ is achieved by both $g$ and $h$ and $2|e|_{\infty}^{\bar{Q}_{L>0}}-|e|_{1}^{\bar{Q}_{L>0}} \leq 0$. Thus

$$
\max \left(0,\left(2|e|_{\infty}^{Q}-|e|_{1}^{Q}\right)\right) \geq \max \left(0,\left(2|e|_{\infty}^{\bar{Q}_{L>0}}-|e|_{1}^{\bar{Q}_{L>0}}\right)\right)
$$


Putting this together we get

$$
\begin{aligned}
r & =\sum_{e \in F_{1}(Z)} f_{1}\left(\pi^{-1} e \cap \partial D\right) \\
& \geq \sum_{e \in F_{1}(Z)} \max \left(0,\left(2|e|_{\infty}^{Q}-|e|_{1}^{Q}\right)\right) \\
& \geq \sum_{e \in F_{1}(Z)} \max \left(0,\left(2|e|_{\infty}^{\bar{Q}_{L>0}}-|e|_{1}^{\bar{Q}_{L>0}}\right)\right) \\
& \geq \sum_{e \in F_{1}(Z)}\left(2|e|_{\infty}^{\left.\bar{Q}_{L>0}-|e|_{1}^{\bar{Q}_{L>0}}\right)}\right. \\
& =\sum_{e \in F_{1}(Z)}\left(\sum_{(i, j) \in \bar{Q}_{L>0}: e \in F_{1}\left(Z_{i, j}\right)}\left(2-f_{e}^{2}\left(Z_{i, j}\right)\right)\right) \\
& =\sum_{(i, j) \in \bar{Q}_{L>0}}\left(\sum_{e \in F_{1}\left(Z_{i, j}\right)}\left(2-f_{e}^{2}\left(Z_{i, j}\right)\right)\right) \\
& =\sum_{(i, j) \in \bar{Q}_{L>0}} L\left(Z_{i, j}\right) .
\end{aligned}
$$

Recall the definition of $K_{A}(Z)$ in Definition 4.14.

Definition 5.7. Define

$$
Z_{L \leq 0}^{\infty}=K_{Z_{L \leq 0}}(Z) \subseteq Z
$$

Similarly set

$$
D_{L \leq 0}^{\infty}=\pi^{-1}\left(Z_{L \leq 0}^{\infty}\right) \subset D
$$

Lemma 5.8. For any filling $(C \stackrel{b}{\rightarrow} D \stackrel{\pi}{\rightarrow} X)$,

$$
L\left(Z_{L \leq 0}\right) \leq 0 \text { and } L\left(Z_{L \leq 0}^{\infty}\right) \leq 0 .
$$

Proof. The $Z_{i, j}$ have a natural tree structure generated by containment. Thus we can write

$$
Z_{L \leq 0}=\bigcup_{(i, j) \in A} Z_{i, j}
$$

where $L\left(Z_{i, j}\right) \leq 0$ for all $(i, j) \in A$. As $L$ is additive on disjoint complexes we get $L\left(Z_{L \leq 0}\right) \leq 0$. By the definition of $L_{L \leq 0}^{\infty}=K_{L \leq 0}(Z)$ every edge

$$
e \in F_{1}\left(Z_{L \leq 0}^{\infty}\right) \backslash F_{1}\left(Z_{L \leq 0}\right)
$$


has $f_{e}^{2}\left(Z_{L \leq 0}^{\infty}\right) \geq 2$. Thus

$$
\begin{aligned}
L\left(Z_{L \leq 0}^{\infty}\right)= & \sum_{e \in F_{1}\left(Z_{L \leq 0}^{\infty}\right)}\left(2-f_{e}^{2}\left(Z^{\prime}\right)\right) \\
\leq & \sum_{e \in F_{1}\left(Z_{L \leq 0}^{\infty} \cap Z_{L \leq 0}\right)}\left(2-f_{e}^{2}\left(Z^{\prime}\right)\right) \\
& +\sum_{e \in F_{1}\left(Z_{L \leq 0}^{\infty}\right) \backslash F_{1}\left(Z_{L \leq 0}\right)}\left(2-f_{e}^{2}\left(Z^{\prime}\right)\right) \\
\leq & L\left(Z_{L \leq 0}\right) \\
\leq & 0 .
\end{aligned}
$$

Lemma 5.9. For every $\epsilon>0$ and 2-dimensional simplicial complex $Z$ with

- $e(Z)>\frac{1}{2}+\epsilon$ and

- $\chi\left(Z^{\prime}\right) \leq 1$ for every connected $Z^{\prime} \subset Z$,

every contractible $\gamma: C_{r} \rightarrow Z$ and minimal filling $\left(C_{r} \stackrel{b}{\rightarrow} D \stackrel{\pi}{\rightarrow} X\right)$ satisfies

$$
f_{2}(D)<\beta r+4 \beta f_{2}\left(D \backslash D_{L \leq 0}\right) .
$$

Proof. By Definition 5.7 we have the complexes $Z_{L \leq 0} \subset Z_{L \leq 0}^{\infty} \subset Z$ and $D_{L \leq 0}^{\infty} \subset D$. Let $\left\{D_{j}\right\}$ be the connected components of $D_{L \leq 0}^{\infty}$. Also define $\gamma_{j}: \partial D_{j} \rightarrow Z$, where $\gamma_{j}(e)=\pi(e)$ and let $r_{j}$ be the length of $\partial D_{j}$. We will now show that every $\gamma_{j}$ is contractible in $Z_{L \leq 0}^{\infty}$, as $\left(C_{r_{j}} \rightarrow D_{j} \stackrel{\left.\pi\right|_{D_{j}}}{\longrightarrow} Z_{L \leq 0}^{\infty}\right)$ gives a filling of $\gamma_{j}$.

Take $d \in D \backslash \bigcup_{j} D_{j}$. Consider $C$, the connected component of $D \backslash \bigcup_{j} D_{j}$ containing $d$. Suppose that $C$ contains no vertex in $b\left(C_{r}\right)$. Let $e$ be an edge in the boundary of $C$. Then by our supposition, $\pi(e) \in Z_{L \leq 0}^{\infty}$. Let $e^{\prime}$ be an edge not in the boundary of $C$. If $f_{e^{\prime}}^{2}\left(\pi(C) \cup Z_{L \leq 0}^{\infty}\right)=1$ and $e^{\prime} \cap b\left(C_{r}\right)=\emptyset$, then we could create a smaller filling by collapsing the two appearances of the face adjacent to $e^{\prime}$ onto a line segment and $\left(C_{r} \stackrel{b}{\rightarrow} D \stackrel{\pi}{\rightarrow} X\right)$ is not minimal. Thus $f_{e^{\prime}}^{2}\left(\pi(C) \cup Z_{L \leq 0}^{\infty}\right) \geq 2$. Combining these two statements about edges in $\pi(C)$ we get that

$$
\pi(C) \subset K_{Z_{L \leq 0}^{\infty}}\left(Z_{L \leq 0}^{\infty}\right)=Z_{L \leq 0}^{\infty} .
$$

This is a contradiction as $C$ was defined to be in $D \backslash D_{L \leq 0}^{\infty}=D \backslash \pi^{-1}\left(Z_{L \leq 0}^{\infty}\right)$. Thus $C$ contains a vertex in $b\left(C_{r}\right)$. This implies that each $\gamma_{j}$ is contractible in $Z_{L \leq 0}^{\infty}$.

By Lemma 5.8 we have that $L\left(Z_{L \leq 0}^{\infty}\right) \leq 0$. By assumption $\chi\left(Z_{L \leq 0}^{\infty}\right) \leq 1$. Thus Lemma 5.2 can be applied and we have that

$$
\beta r_{j}=\beta\left|\partial D_{j}\right|>A\left(\gamma_{j}\right) .
$$

By the definition of the $D_{j}$, every edge in $\bigcup \partial D_{j}$ is either in the boundary of $D$ or adjacent to a face in $D \backslash D_{L \leq 0}^{\infty}$. Thus

$$
f_{1}\left(\partial D_{L \leq 0}^{\infty}\right)=\sum_{j} f_{1}\left(\partial D_{j}\right)=\sum_{j} r_{j} \leq r+3 f_{2}\left(D \backslash D_{L \leq 0}^{\infty}\right)
$$

or

$$
r \geq \sum r_{j}-3 f_{2}\left(D \backslash D_{L \leq 0}^{\infty}\right) .
$$


By the definition of $Z_{L \leq 0}^{\infty}$ we have $Z_{L \leq 0} \subset Z_{L \leq 0}^{\infty}$, so

$$
f_{2}\left(D \backslash D_{L \leq 0}^{\infty}\right) \leq f_{2}\left(D \backslash D_{L \leq 0}\right) .
$$

Thus multiplying (13) by $\beta$ we get

$$
\begin{aligned}
\beta r & \geq \sum \beta r_{j}-3 \beta f_{2}\left(D \backslash D_{L \leq 0}^{\infty}\right) \\
& >\sum A\left(\gamma_{j}\right)-3 \beta f_{2}\left(D \backslash D_{L \leq 0}^{\infty}\right) \\
& \geq f_{2}(D)-f_{2}\left(D \backslash D_{L \leq 0}^{\infty}\right)-3 \beta f_{2}\left(D \backslash D_{L \leq 0}^{\infty}\right) \\
& \geq f_{2}(D)-4 \beta f_{2}\left(D \backslash D_{L \leq 0}^{\infty}\right) \\
& \geq f_{2}(D)-4 \beta f_{2}\left(D \backslash D_{L \leq 0}\right),
\end{aligned}
$$

which proves the lemma.

Lemma 5.10. For every $\epsilon>0$ and simplicial complex $X$ with $e(X)>\frac{1}{2}+\epsilon$, contractible $\gamma: C_{r} \rightarrow X$ and minimal filling $(C \stackrel{b}{\rightarrow} D \stackrel{\pi}{\rightarrow} X)$,

$$
f_{2}\left(D \backslash D_{L \leq 0}\right)<\frac{3}{2 \epsilon} r
$$

Proof. We use Lemma 5.1 with $w=0$ to get that for each $(i, j) \in \bar{Q}_{L>0}$,

$$
L\left(Z_{i, j}\right)+2 \chi\left(Z_{i, j}\right) \geq f_{2}\left(Z_{i, j}\right)\left(2 e\left(Z_{i, j}\right)-1\right) .
$$

By assumption we have that $\chi\left(Z_{i, j}\right) \leq 1, L\left(Z_{i, j}\right) \geq 1$, and $e\left(Z_{i, j}\right)>\epsilon$, so

$$
\begin{aligned}
3 L\left(Z_{i, j}\right) & \geq L\left(Z_{i, j}\right)+2 \\
& \geq L\left(Z_{i, j}\right)+2 \chi\left(Z_{i, j}\right) \\
& >2 \epsilon f_{2}\left(Z_{i, j}\right) .
\end{aligned}
$$

Thus by Lemma 5.6 .

$$
\begin{aligned}
3 r & \geq \sum_{Q_{L>0}} 3 L\left(Z_{i, j}\right) \\
& \geq \sum_{Q_{L>0}} 2 \epsilon f_{2}\left(Z_{i, j}\right) \\
& =2 \epsilon f_{2}\left(D \backslash D_{L \leq 0}\right) .
\end{aligned}
$$

Proof of Lemma 3.5. If $A(\gamma)=\infty$, then we are done. If not, then $\gamma$ is contractible in $X$. By Lemma 4.1 we can find a subcomplex $Z \subset X$ such that $\chi\left(Z^{\prime}\right) \leq 1$ for all connected $Z^{\prime} \subset Z$ and $\gamma$ is contractible in $Z$. Let $(C \stackrel{b}{\rightarrow} D \stackrel{\pi}{\rightarrow} Z)$ be a minimal filling in $Z$. By the definition of $e$ we have

$$
e(Z) \geq e(X) \geq \frac{1}{2}+\epsilon,
$$

so the hypotheses of Lemmas 5.9 and 5.10 apply. Thus we can apply Lemmas 5.9 and 5.10 to $\gamma$ and $Z$. The area of $\gamma$ in $X$ is at most the area of $\gamma$ in $Z$, so

$$
A_{X}(\gamma) \leq A_{Z}=f_{2}(D)
$$


Combining Lemmas 5.9 and 5.10 , we get

$$
\begin{aligned}
A_{X}(\gamma) & \leq f_{2}(D) \\
& \leq \beta r+4 \beta f_{2}\left(D \backslash D_{L \leq 0}\right) \\
& \leq \beta r+4 \beta \frac{3}{2 \epsilon} r \\
& <\beta r+\frac{6}{\epsilon} \beta r \\
& <\frac{7 \beta}{\epsilon} r .
\end{aligned}
$$

Proof of Lemma 3.11, If $\operatorname{Id}_{[3]}$ is contractible in $X$, then by Lemma 4.1 there exists $Z \subset X$ such that $\chi\left(Z^{\prime}\right) \leq 1$ for all connected $Z^{\prime} \subset Z$ and $\operatorname{Id}_{[3]}$ is contractible in $Z$. Let $(C \stackrel{b}{\rightarrow} D \stackrel{\pi}{\rightarrow} Z)$ be a minimal filling of $\operatorname{Id}_{[3]}$ in $Z$. Define $Z^{\prime}$ by $F_{2}\left(Z^{\prime}\right)=$ $\pi\left(F_{2}(D)\right)$. Thus $\operatorname{Id}_{[3]}$ is a contractible 0 -immersion in $Z^{\prime}$ and by Lemma [5.4, we have that $(C \stackrel{b}{\rightarrow} D \stackrel{\pi}{\rightarrow} Z)$ is a 1 -immersion. Thus

$$
L\left(Z^{\prime}\right) \leq L(D) \leq 3 .
$$

By Lemma 5.1 we have that

$$
\begin{aligned}
f_{2}\left(Z^{\prime}\right) & \leq \frac{2 \chi\left(Z^{\prime}\right)-2 \cdot 3+L\left(Z^{\prime}\right)}{e_{3}\left(Z^{\prime}\right)} \\
& \leq \frac{2 \cdot 1-2 \cdot 3+3}{e_{3}(X)} \\
& <0 .
\end{aligned}
$$

This is a contradiction and $\operatorname{Id}_{[3]}$ is not contractible in $Z^{\prime}$ or in $X$.

\section{Open PROBLEMS}

Various kinds of random finitely presented groups have been studied by geometric group theorists. We refer the interested reader to 12 for a very nice survey and introduction. One particular model of a random group seems closely related to $\pi_{1}(Y(n, p))$. Let $b_{1}, \ldots, b_{n}$ be $n$ distinct symbols, and let $W$ be the set of reduced words of length 3 in $\left\{b_{1}^{ \pm 1}, \ldots, b_{n}^{ \pm 1}\right\}$.

Definition 6.1. Let $0 \leq d \leq 1$. A triangular random group on $n$ relators at density $d$ is the group presented by $H=\left\langle b_{1}, \ldots, b_{n} \mid R\right\rangle$, where $R$ is a set of $|W|^{d}$ words chosen at random uniformly from $W$.

As before we say that $H$ a.a.s. has property $\mathcal{P}$ if $\mathbf{P}(H \in \mathcal{P}) \rightarrow 1$ as $n \rightarrow \infty$. The main results about triangular random groups are the following.

Theorem 6.2 ([15]). If $d<1 / 2$, then $H$ is a.a.s. nontrivial hyperbolic, and if $d>1 / 2$, then $H$ is a.a.s. trivial.

This is analogous to our main result, although there are noteworthy differences. For instance, in the triangular model, $|W|$ is approximately $8 n^{3}$, so at the threshold the number of relators is roughly the number of generators raised to the $3 / 2$ power. But at the threshold for $\pi_{1}(Y)$, the number of relators is roughly the number of generators to the $5 / 4$ power. 
Theorem 6.3 ([15]). If $d<1 / 3$, then $H$ is a.a.s. free. If $d>1 / 3$, then $H$ a.a.s. has property $(T)$.

Kazhdan's property $(T)$ is a condition usually stated in terms of unitary representations. See [15] for a nice formulation of Property $(T)$ for discrete groups.

This second theorem of Żuk's seems to be somewhat analogous to the LinialMeshulam threshold for homology. In both cases, the number of generators is roughly equal to the number of relators. It might be reasonable to expect that when $p \ll n^{-1}, \pi_{1}(Y)$ is a.a.s. free, and that when $p \gg n^{-1}, \pi_{1}(Y)$ a.a.s. has property $(T)$, but at the present moment we do not know either of these as facts.

Similarly, it follows from property $(T)$ that the triangular random groups have finite abelianizations when $d>1 / 3$. One might expect that these abelianizations are in fact trivial, but it seems that this is not known.

Similar comments should also hold for $H_{1}(Y, \mathbb{Z})$. If $H_{1}(X, \mathbb{Z} / p \mathbb{Z})=0$ for every $p$, then $H_{1}(X, \mathbb{Z})=0$ as well [7. By the Linial-Meshulam-Wallach results ([10, 11]), $H_{1}(Y, \mathbb{Z})$ is finite and has no $p$-torsion for any fixed $p$. So once $p \gg 2 \log n / n$, either $H_{1}(Y, \mathbb{Z})$ is trivial, or it is a finite generated abelian group with torsion approaching infinity. The first scenario might seem more plausible, but as far as we know, nothing is proved either way.

Finally, Friedgut and Kalai's theorem on sharp thresholds [4 holds fairly generally and would seem to imply that there is a sharper threshold for simple connectivity than what is shown here. It would be nice to know the location and "width" of the threshold more precisely, and the behavior of $\pi_{1}(Y(n, p))$ within the critical window would be especially interesting to know. Itai Benjamani asks: if we think of $Y(n, p)$ as a stochastic process where the random triangles are added one at a time, what can be said about the last nontrivial $\pi_{1}$ ?

\section{ACKNOWLEDGEMENTS}

The authors thank Nati Linial, Roy Meshulam, and Omer Angel for helpful conversations. We also acknowledge Rick Kenyon for mentioning the work of Ollivier and the connection to Gromov's theory of random groups. We would especially like to thank an anonymous referee for a careful reading of an earlier draft of this paper and for several helpful remarks.

\section{Appendix 1. Connectivity of $G(n, p)$ AWAY From the threshold}

Proof of Lemma 2.2. We show that if $p=\frac{3 \log n+c}{n}$, then the probability that the graphs $\operatorname{lk}_{Y}(a) \cap \mathrm{lk}_{Y}(b)$ are connected for all pairs $\{a, b\}$ is bounded below by $1-C e^{-c}$ with $C$ independent of $c$. Since the probability that $T Y(a, b)$ is connected for all $a$ and $b$ in $[n]$ is increasing in $p$ this is enough to prove that the condition occurs a.a.s. These methods are typical in random graph theory; see for example [1, Theorem 7.3.

If $G$ is a graph with $n-2$ vertices and with no connected components consisting of $k$ vertices, then $G$ is connected.

For $k$ between 1 and $n$, let $E_{k}$ be the expected number of connected components in $T Y(a, b)$ with $k$ vertices. We will show that

$$
\sum_{a, b} \sum_{k=1}^{\lfloor n / 2\rfloor} E_{k}=\left(\begin{array}{l}
n \\
2
\end{array}\right) \sum_{k=1}^{\lfloor n / 2\rfloor} E_{k} \leq C e^{-c}
$$


for some $C<\infty$. Thus by the union bound and the remark above, all $T Y(a, b)$ are connected a.a.s.

For any set of vertices $\{a, b, u, v\}, u$ is adjacent to $v$ in $T Y(a, b)$ if and only if $\{a, u, v\}$ and $\{b, u, v\}$ are both faces of $Y$, which happens with probability $p^{2}$ by independence. So the probability that $x$ is an isolated vertex in $T Y(a, b)$ is $\left(1-p^{2}\right)^{n-3}$, and we have that

$$
\begin{aligned}
E_{1} & =(n-2)\left(1-p^{2}\right)^{n-3} \\
& =(n-2)\left(1-\frac{3 \log n+c}{n}\right)^{n-3} \\
& <C(n-2) e^{-3 \log n+c} \\
& <C e^{-c} / n^{2} \\
& <C e^{-c}
\end{aligned}
$$

for some constant $C<\infty$.

The expected number of connected components in $T Y(a, b)$ of order 2 is

$$
\begin{aligned}
E_{2} & <\left(\begin{array}{c}
n-2 \\
2
\end{array}\right) p^{2}\left(1-p^{2}\right)^{2(n-4)} \\
& <n^{2}\left(\frac{3 \log n+c}{n}\right)^{2}\left(1-\frac{3 \log n+c}{n}\right)^{2(n-4)} \\
& <C n^{2} e^{-2(3 \log n+c)} \\
& <C e^{-2 c} / n^{4} \\
& <C e^{-c} .
\end{aligned}
$$

Similarly, since the number of spanning trees on a fixed set of $k$ vertices is $k^{k-2}$,

$$
\begin{aligned}
\sum_{a, b} \sum_{k=3}^{\lfloor n / 2\rfloor} E_{k} & \leq\left(\begin{array}{c}
n \\
2
\end{array}\right) \sum_{k=3}^{\lfloor n / 2\rfloor} E_{k} \\
& \leq\left(\begin{array}{c}
n \\
2
\end{array}\right) \sum_{k=3}^{\lfloor n / 2\rfloor}\left(\begin{array}{c}
n-2 \\
k
\end{array}\right) k^{k-2} p^{2(k-1)}\left(1-p^{2}\right)^{k(n-k-2)} \\
& \leq\left(\frac{n^{2}}{2}\right) \sum_{k=3}^{\lfloor n / 2\rfloor} \frac{n^{k}}{k !} k^{k-2} p^{2(k-1)} e^{-p^{2} k(n-k-2)} \\
& \leq\left(\frac{n^{2}}{2}\right) \sum_{k=3}^{\lfloor n / 2\rfloor} k^{-5 / 2} e^{k} n^{k} p^{2(k-1)} e^{-p^{2} k(n-k-2)} \\
& \leq\left(\frac{n^{3}}{2}\right) \sum_{k=3}^{\lfloor n / 2\rfloor} k^{-5 / 2} \exp [k+(k-1) \log 3+(k-1) \log \log n \\
& \leq\left(\frac{n^{3}}{2}\right) \sum_{k=3}^{\lfloor n / 2\rfloor} k^{-5 / 2} \exp [-7 k \log n / 5] \\
& \leq n^{-6 / 5} \\
& <C e^{-c} .
\end{aligned}
$$


For the second condition, note that for fixed $a, b, d \in[n]$ we have that

$$
\mathbf{P}\left(a b d \notin F_{2}(Y)\right)=1-p .
$$

For each $d$ this is independent. So for a fixed $a, b \in[n]$,

$$
\mathbf{P}\left(\nexists d: a b d \in F_{2}(Y)\right)=(1-p)^{n-2}=O\left(1-\frac{1}{n^{1 / 2}}\right)=O\left(e^{-n^{1 / 2}}\right) .
$$

Then the union bound shows that the second condition is satisfied a.a.s.

\section{APPENDIX 2. LOCAL-TO-GLOBAL}

In this appendix we prove a local-to-global theorem for linear isoperimetric inequalities. The statement and proof are similar in spirit to results already appearing for groups (see [5, 13]), but we need the result for simplicial complexes, so we include a proof here for the sake of completeness.

Throughout the section we fix $\epsilon \in(0, .25)$ and work with simplicial complexes scaled so that edges have length $\epsilon$ and triangles have area $\epsilon^{2}$.

Theorem 6.4. If $X$ is a simplicial complex with edge lengths $\epsilon$ and triangle areas $\epsilon^{2}$ and there is an $n \geq 1$ such that every loop $\gamma$ with $1 \leq A \gamma \leq 44$ has $A \gamma<\left(\frac{L \gamma}{44}\right)^{n}$, then every contractible loop $\gamma$ with $1 \leq A \gamma$ has $A \gamma<L \gamma$.

Theorem 3.9 follows easily from this result. The key concept that we will use in the proof of Theorem 6.4 is that of a shortcut.

Definition 6.5. We define $I_{r}$ to be the path of length $r$ with $F_{0}\left(C_{r}\right)=[r]=$ $\{1, \ldots, r\}([0]=\emptyset)$ and

$$
F_{1}\left(C_{r}\right)=\bigcup_{i=1}^{r-1}\{\{i, i+1\}\} \cup\{r, 1\}
$$

Definition 6.6. Let $F=(C \stackrel{b}{\rightarrow} D \stackrel{\pi}{\rightarrow} X)$ be a filling. A $k$-marking of $F$ is

$$
R=([k] \stackrel{x}{\rightarrow} C \stackrel{b}{\rightarrow} D \stackrel{\pi}{\rightarrow} X)
$$

where $[k] \stackrel{x}{\rightarrow} C$ is a cyclically order-preserving map from $[k]$ to $F_{0}(C)=[L C]$. (This simply means that there exists $a \in[k]$ so that $x_{i}<x_{i+1}$ if $i \neq a$ and $x_{k}<x_{1}$ if $k \neq a$.) For each marked filling we define the covering by cyclically order-preserving paths $J_{i}: I_{x_{i+1}-x_{i}} \rightarrow C$ from $x_{i}$ to $x_{i+1}$ and $J_{k}: I_{x_{1}-x_{k}+L C} \rightarrow C$ from $x_{k}$ to $x_{1}$. Define paths $b_{i}=b\left(J_{i}\right): I \rightarrow D$ and $\gamma_{i}=\pi\left(b_{i}\right): I \rightarrow X$.

Definition 6.7. A shortcut is a 2-marked filling

$$
S=([2] \stackrel{x}{\rightarrow} C \stackrel{b}{\rightarrow} D \stackrel{\pi}{\rightarrow} X)
$$

with

$$
d\left(x_{1}, x_{2}\right)-d\left(b x_{1}, b x_{2}\right) \geq 1 .
$$

Fix a path $B: I_{d\left(b x_{1}, b x_{2}\right)} \rightarrow D$ from $b x_{1}$ to $b x_{2}$.

We say that a shortcut is of type $\mu$ if

$$
d\left(x_{1}, x_{2}\right) \geq \mu \quad \text { and } \quad d\left(b x_{1}, b x_{2}\right) \leq \mu-1 .
$$

Note that every shortcut has at least one type.

We prove Theorem 6.4 by induction on the area of the filling.

Throughout the rest of this section we let $\alpha=44$ and $\mu=\frac{13}{2}$. The first step is to show that all shortcuts in a minimal counterexample are long. 
Lemma 6.8. Let

$$
F=(C \stackrel{b}{\rightarrow} D \stackrel{\pi}{\rightarrow} X)
$$

be a filling which is a minimal area counterexample to Theorem 6.4, Every shortcut $\gamma$ in $F$ has length and area at least $\alpha$.

Proof. Take $([2] \stackrel{x}{\rightarrow} C \stackrel{b}{\rightarrow} D \stackrel{\pi}{\rightarrow} X)$ to be a shortcut in $F$. Then we have three paths $b_{1}, \overline{b_{2}}, B$ from $b x_{1}$ to $b x_{2}$ and may assume that

$$
L B+1<L b_{1} \leq L b_{2} .
$$

Denote the cycles obtained by first traversing $b_{i}$ forward and the $B$ backward by $B_{i}=b_{i} \cdot \bar{B}$. Denote the induced cycles in $X$ by $\gamma_{i}=\pi B_{i}$. Note that $A \gamma_{i}=A B_{i}$.

First note that $\alpha<A \gamma$. Otherwise, as $\gamma$ is a counterexample to Theorem 6.4 and $A \gamma<\alpha$,

$$
L \gamma \leq A \gamma<\left(\frac{L \gamma}{\alpha}\right)^{n}
$$

So

$$
\alpha<\alpha^{\frac{n}{n-1}}<L \gamma \leq A \gamma
$$

which is a contradiction.

Note that $1 \leq A \gamma_{1}$. Otherwise,

$$
A \gamma_{2}=A \gamma-A \gamma_{1}>\alpha-1 \geq 1 \text {. }
$$

Since $\gamma$ is a minimal area counterexample we must have that $\gamma_{i}$ is not a counterexample, so $A \gamma_{i}<L \gamma_{i}$. Hence by the definitions of the $\gamma_{i}$ and of a shortcut,

$$
1>A \gamma_{1}=A \gamma-A \gamma_{2}>L \gamma-L \gamma_{2}=L J_{1}-L B=d\left(x_{1}, x_{2}\right)-d\left(b x_{1}, b x_{2}\right) \geq 1,
$$

a contradiction.

Finally, if $L \gamma_{1} \leq \alpha$, then

$$
1 \leq A \gamma_{1}<L \gamma_{1} \leq \alpha
$$

so that by the hypotheses of Theorem 6.4, $1 \leq A \gamma_{1}<\left(\frac{L \gamma_{1}}{\alpha}\right)^{n}$ and $\alpha<L \gamma_{1}$, a contradiction.

Definition 6.9. A rectangle of type $\left(u_{1}, u_{2}\right)$ is a 4 -marked filling $R$ with

$$
d\left(b_{i}, b_{i+2}\right) \geq u(i)
$$

for both $i=1,2$. Write $A(R)=A(D)$ for the area.

Definition 6.10. The ball $B(r, A)$ of radius $r$ about $A \subseteq X$ (a metric space) is all points with distance to $A$ at most $r$.

Definition 6.11. If $R$ is a rectangle with type $u_{j}>r$, then the $r$-neighborhood of the $i=j$ (or $i=j+2$ ) edge of $R$ is the subrectangle

$$
N_{r, i}(R)=\left([4] \stackrel{x^{\prime}}{\rightarrow} C^{\prime} \stackrel{b^{\prime}}{\rightarrow} D^{\prime} \stackrel{\left.\pi\right|_{D^{\prime}}}{\longrightarrow} X\right) .
$$

Take $y_{1}, y_{2} \in b^{-1} B\left(r, \operatorname{Im}\left(b_{i}\right)\right)$ maximizing the length $t$ of the path $a: I_{t} \rightarrow C$ from $y_{1}$ to $y_{2}$ containing $\operatorname{Im}\left(J_{i}\right)$ but not intersecting $\operatorname{Im}\left(J_{i \pm 2}\right)$. Take $x_{i}^{\prime}=x_{i}$, $x_{i+1}^{\prime}=x_{i+1}, x_{i \pm 2}^{\prime}=y_{1}$ and $x_{i+1 \pm 2}^{\prime}=y_{2}$. Take $z: I_{s} \rightarrow D$ to be the 1-immersed path from $b y_{2}$ to $b y_{1}$ with interior avoiding $\operatorname{Im}(b)$ along the boundary (interior) of $B\left(r, \operatorname{Im}\left(b_{i}\right)\right)$. Take $b^{\prime}=a \cdot \bar{z}: C^{\prime}=C_{t+s} \rightarrow D^{\prime} \subseteq D$. 
Lemma 6.12. If $R$ is a rectangle of type $(r+s, v)$, then $N_{r, 1}(R)$ is of type $(r, v)$ and has interior disjoint from $N_{s, 3}(R)$.

Proof. If there is a point in $N_{r, 1}(R)^{\circ} \cap N_{s, 3}(R)^{\circ}$, then there is a path from $\operatorname{Im}\left(b_{1}\right)$ to $\operatorname{Im}\left(b_{3}\right)$ of length less than $r+s$.

Lemma 6.13. If $R$ is a rectangle of type $u$, then $A(R) \geq 2 u(1) u(2)$.

Proof. Since any type $(\epsilon, \epsilon)$ rectangle has at least 2 triangles, an easy induction using Lemma 6.12 now shows that any type $(u(1), u(2))$ rectangle has area at least $2 u(1) u(2)$.

Lemma 6.14. Let

$$
F=(C \stackrel{b}{\rightarrow} D \stackrel{\pi}{\rightarrow} X)
$$

be a minimal filling which is a minimal area counterexample to Theorem 6.4. Then there exists a filling

$$
F^{\prime}=\left(C^{\prime} \stackrel{b^{\prime}}{\rightarrow} D^{\prime} \stackrel{\left.\pi\right|_{D^{\prime}}}{\longrightarrow} X\right)
$$

with $A \gamma \geq A \gamma^{\prime}>\alpha$ and $A \gamma^{\prime}>1.15 L \gamma^{\prime}$.

Proof. In the proof we perform the following steps.

Define the filling $F^{\prime}$ and a marking $r$. Write $\mu \leq m \epsilon<\mu+\epsilon$.

We do this in two cases. First if $F$ has no type $\mu$ shortcut, then our filling $F^{\prime}=F$. Define a $2 t$-marking with $r_{i}=1+m(i-1)$ and $t$ maximal with $L C \geq 2 t m \epsilon$.

If $F$ does have a type $\mu$ shortcut, then choose one from $x_{1}$ to $x_{2}$ such that $L J_{1}$ is minimal (and among those, choose one with $A B_{1}$ minimal). Our filling $F^{\prime}$ will have $b^{\prime}=b_{1} \cdot \overline{b_{3}}$.

Now we define a $(2 t)$-marking of $F^{\prime}$ by $r_{i}=x_{1}+m i \in \operatorname{Im}\left(J_{1}\right)$, taking $t$ maximal so that $m \epsilon(2 t+1) \leq L J_{1}$. Note that all of the paths $b_{i}$ for $i=1, \ldots, 2 t-1$ for this marked filling have image in that of $b_{1}$ for the marked filling $x$. Note that in both cases,

$$
t>\frac{L J_{1}-(\mu-1)-2(\mu+\epsilon)}{2(\mu+\epsilon)} .
$$

Define the rectangles $R_{i}$.

For any $i<j \in[t]$ consider the type $\left(u_{1}=\mu-1, u_{2}=\mu-1\right)$ rectangle $R_{i, j}$ marking $F^{\prime}$ by $y_{1}=r_{i}, y_{2}=r_{i+1}, y_{3}=r_{j}$ and $y_{4}=r_{j+1}$. The type follows from the minimality of the shortcut and Lemma 6.12. Define $R_{i}=N_{\frac{\mu-1}{2}, 1}\left(R_{2 i, 2 j}\right)=$ $N_{\frac{\mu-1}{2}, 3}\left(R_{2 k, 2 i}\right)=N_{\frac{\mu-1}{2}, 2}\left(R_{i-1, i+1}\right)$. By Lemma 6.12 $R_{i}$ has type $u_{1}=\frac{\mu-1}{2}, u_{2}=\mu$ and has interior disjoint from $R_{j}$.

$A\left(R_{i}\right)>(\mu-1)^{2}$ for each $i$.

By Lemma 6.13 and the last step we have that

$$
A\left(R_{i}\right) \geq 2\left(\frac{\mu-1}{2}\right)(\mu-1) \geq(\mu-1)^{2} .
$$


Finally we compute the area of $b$.

Recalling that $\mu=6.5, L b^{\prime}>44$ and $\epsilon<.1$, we have that $(\mu-1)^{2}=30.25$ and

$$
\begin{aligned}
A b^{\prime} & \geq \sum_{i=1}^{t} A\left(R_{i}\right) \geq t(\mu-1)^{2} \\
& \geq\left(\frac{L b^{\prime}-3 \mu-2 \epsilon+1}{2(\mu+\epsilon)}\right)(\mu-1)^{2} \geq \frac{30.25 L b-575}{14}>2.15 L b^{\prime}-44>1.15 L B^{\prime} .
\end{aligned}
$$

Proof of Theorem 6.4. Assume not. Fix a counterexample $\gamma: C \rightarrow X$ with $1 \leq L \gamma \leq A \gamma$ such that $A \gamma$ is minimal as well as a minimal filling

$$
F=(C \stackrel{b}{\rightarrow} D \stackrel{\pi}{\rightarrow} X)
$$

of $\gamma$.

By Lemma 6.14 we have that $A \gamma>1.25 L \gamma$. We construct a smaller counterexample. Simply alter $D$ by removing one 2 -face touching the boundary of $D$ and make the corresponding changes to the rest of the filling. This increases the length of the curve by at most $\epsilon<.25$ and decreases its area by at most $\epsilon^{2}<.0625$. It is easy to check that this is still a counterexample. As $\gamma$ was minimal, this is a contradiction.

Proof of Theorem 3.9. Rescale $X$ so that edges have length $\frac{44}{\rho}$ and triangles have area $\left(\frac{44}{\rho}\right)^{2}$ and apply Theorem 6.4 with $n=1$.

Theorem 6.4 is closely related to the gap between quadratic and linear growth, as discussed for instance in 2]. In fact this gap follows from Theorem 6.4 with $n=2$.

\section{REFERENCES}

[1] Béla Bollobás. Random graphs, volume 73 of Cambridge Studies in Advanced Mathematics. Cambridge University Press, Cambridge, second edition, 2001. MR1864966 (2002j:05132)

[2] B. H. Bowditch. A short proof that a subquadratic isoperimetric inequality implies a linear one. Michigan Math. J., 42(1):103-107, 1995. MR1322192 (96b:20046)

[3] P. Erdős and A. Rényi. On random graphs. I. Publ. Math. Debrecen, 6:290-297, 1959. MR.0120167 (22:10924)

[4] Ehud Friedgut and Gil Kalai. Every monotone graph property has a sharp threshold. Proc. Amer. Math. Soc., 124(10):2993-3002, 1996. MR.1371123(97e:05172)

[5] M. Gromov. Hyperbolic groups. In Essays in group theory, volume 8 of Math. Sci. Res. Inst. Publ., pages 75-263. Springer, New York, 1987. MR919829 (89e:20070)

[6] M. Gromov. Asymptotic invariants of infinite groups. In Geometric group theory, Vol. 2 (Sussex, 1991), volume 182 of London Math. Soc. Lecture Note Ser., pages 1-295. Cambridge Univ. Press, Cambridge, 1993. MR.1253544 (95m:20041)

[7] Allen Hatcher. Algebraic topology. Cambridge University Press, Cambridge, 2002. MR:1867354 (2002k:55001)

[8] Matthew Kahle. The neighborhood complex of a random graph. J. Combin. Theory Ser. A, 114(2):380-387, 2007. MR2293099 (2008a:05247)

[9] Matthew Kahle. Topology of random clique complexes. Discrete Math., 309(6):1658-1671, 2009. MR2510573 (2010h:05276)

[10] Nathan Linial and Roy Meshulam. Homological connectivity of random 2-complexes. Combinatorica, 26(4):475-487, 2006. MR 2260850(2007i:55004)

[11] R. Meshulam and N. Wallach. Homological connectivity of random $k$-dimensional complexes. Random Structures Algorithms, 34(3):408-417, 2009. MR2504405 (2010g:60015) 
[12] Yann Ollivier. A January 2005 invitation to random groups, volume 10 of Ensaios Matemáticos [Mathematical Surveys]. Sociedade Brasileira de Matemática, Rio de Janeiro, 2005. MR2205306 (2007e:20088)

[13] P. Papasoglu. An algorithm detecting hyperbolicity. In Geometric and computational perspectives on infinite groups (Minneapolis, MN and New Brunswick, NJ, 1994), volume 25 of DIMACS Ser. Discrete Math. Theoret. Comput. Sci., pages 193-200. Amer. Math. Soc., Providence, RI, 1996. MR.1364185 (96k:20075)

[14] Nicholas Pippenger and Kristin Schleich. Topological characteristics of random triangulated surfaces. Random Structures Algorithms, 28(3):247-288, 2006. MR2213112 (2007d:52019)

[15] A. Żuk. Property (T) and Kazhdan constants for discrete groups. Geom. Funct. Anal., 13(3):643-670, 2003. MR1995802 (2004m:20079)

Department of Mathematics, University of California at Davis, Davis, California 95616

E-mail address: babson@math.ucdavis.edu

Department of Mathematics, University of Washington, Seattle, Washington 98195

E-mail address: hoffman@math. washington.edu

Department of Mathematics, Stanford University, Stanford, California 94305

E-mail address: kahle@math.stanford.edu 